\title{
Inflación y crecimiento a largo plazo: malas predicciones, ¿buenos modelos?
}

\author{
Santiago Javier FuCCI* \\ Martín Grandes
}

\begin{abstract}
RESUMEN
Es un hecho bien documentado por la teoría económica que la inflación alta y duradera tiene un efecto negativo en el crecimiento económico, pero que a medida que desciende los efectos sobre este último son no lineales. Este trabajo responde dos preguntas de investigación relacionadas a los trabajos que han estudiado esta relación empírica en las últimas tres décadas. La primera es sobre su validez externa: ¿Qué tan útiles son para predecir la evolución económica de los países emergentes? Simulando el crecimiento de una muestra de mercados emergentes para los últimos veinte ańos a partir de esos modelos, encontramos errores de predicción elevados y dispares y por ende ciertos inconvenientes a la hora de generalizar sus conclusiones. Entender por qué sucede esto da lugar a la segunda pregunta, vinculada a la validez interna de los modelos: ¿Qué problemáticas no resueltas existen en las estimaciones llevadas a cabo por los autores? la respuesta es que existen problemas de especificación, de simultaneidad y de omisión de variables, los cuales generan endogeneidad y entorpecen la identificación causal del efecto de la inflación en el crecimiento en el tipo de regresiones bajo análisis, aún en modelos con umbrales y no lineales.
\end{abstract}

Palabras clave: inflación, crecimiento económico, modelos macroeconómicos.

Clasificación JEL: C52, C53, O47, E31, O49

\section{Inflation and long term growth: bad predictions, good models?}

\begin{abstract}
It is a fact well documented by economic theory that high and durable inflation has a negative effect on economic growth, but that as the effects on the latter decline, they are non-linear. This work answers two research questions related to the works that have studied this empirical

* Docente auxiliar de Crecimiento Económico y Cuentas Nacionales en la Universidad de Buenos Aires. Especialista en tópicos de econometría y en data mining aplicado al sector financiero. Teléfono: 549 0116013 3390. e-mail: sj.fucci@gmail.com Domicilio/Oficina: Simbrón 4776, Ciudad de Buenos Aires.

** Doctor en Economía por la Ecole des Hautes Etudes en Sciences Sociales de Paris (2004). Investigador del CONICET. Docente titular en la Universidad de Buenos Aires, Universidad Católica Argentina y en la Escuela de Gobierno UTDT. Profesor invitado en otras universidades de Francia, Estados Unidos, Colombia, México y China. Senior Fulbright Researcher 2012. Domicilio/Oficina: Avenida Córdoba 2122, Piso 1, Ciudad de Buenos Aires.
\end{abstract}


relationship in the last three decades. The first is about its external validity: How useful are they to predict the economic evolution of emerging countries? Simulating the growth of a sample of emerging markets for the past 20 years from these models, we found high and disparate prediction errors and therefore certain drawbacks when generalizing their conclusions. Understanding why this happens gives rise to the second question, linked to the internal validity of the models: What unresolved problems exist in the estimates carried out by the authors? the answer is that there are problems of specification, simultaneity and omission of variables, which generate endogeneity and hinder the causal identification of the effect of inflation on growth in the type of regressions under analysis, even in models with thresholds and non-linear.

Keywords: inflation, economic growth, macroeconomic modelling.

JEL Classification: C52, C53, O47, E31, O49

\section{INTRODUCCIÓN}

La cuestión relevante no es si [las investigaciones] pueden darnos respuestas definitivas, es si pueden modificar nuestras preconcepciones

Dani Rodrik, 2008.

Desde la posguerra hasta nuestros días, la ciencia económica ha estudiado extensamente los efectos a largo plazo de la inflación en el crecimiento económico. Este tópico es de interés no sólo a nivel teórico, sino que además es y ha sido de actualidad en economías como la argentina y venezolana u otras latinoamericanas en la década de $1980^{1}$. Argentina y Venezuela, particularmente, llevan más de una década inmersas en un ciclo de inflación moderada a alta y se encuentran, en el caso de Argentina, en un proceso de estabilización (cuyo resultado final aún es incierto a 2017), en un contexto de varios años de estancamiento del PBI (Vegh et al., 2017).

La inflación es un fenómeno con múltiples causas, y cuyos efectos en la economía presentan una gran complejidad. Asimismo, no es un fenómeno que opere de forma aislada, sino que tiene un complejo entramado de interacciones con otros aspectos, como la sostenibilidad fiscal y el régimen cambiario, dentro de un concepto más amplio que podríamos denominar estabilidad macroeconómica (Fischer, 1993; Fanelli y Frenkel, 1996)

Más allá de la curva de Philips de corto plazo que relaciona inversamente al nivel de actividad y la tasa de inflación, la estabilidad macroeconómica, en general, y la inflación, en particular, podrían tener implicancias para la performance de largo plazo de las economías. Concretamente, la tasa de crecimiento del producto puede verse afectada por la

\footnotetext{
1 Véase por ejemplo Devlin y Ffrench-Davis (1994) o Fanelli y Frenkel (1996), entre otros.
} 
persistencia de un marco macroeconómico inestable caracterizado específicamente por una inflación alta y duradera.

A grandes rasgos, pueden discriminarse tres amplias corrientes teóricas que explican dicha relación de largo plazo: aquellos autores que sostienen que no existe tal vínculo de largo plazo entre ambas variables (Sidrawski, 1967), aquellos que sostienen que existe un efecto positivo de la inflación en el crecimiento económico (Tobin, 1965; Thirwall y Barton, 1971), y aquellos que defienden la existencia de un efecto negativo (Stockman, 1981; Choi, Smith y Boyd, (1996), o inclusive negativo a partir de cierto umbral para la tasa de inflación (Easterly y Bruno, 1998; Moreno-Brid et al., 2014) comprendiendo modelos del tipo de crecimiento endógeno (Jones y Manuelli, 1995). Lucas (1973), ubicándose en el mediano plazo, explica que para decir algo respecto al impacto de la inflación en el crecimiento económico es necesario previamente saber el tipo de economía bajo análisis: cuando los agentes están expuestos a una inflación alta y volátil, cambios en ésta no tendrán efecto en sus decisiones reales. En cambio, en países estables y sin tradición inflacionaria, la inflación puede afectar la tasa de crecimiento a través de un relajamiento de las rigideces nominales de la economía.

Por el lado de la investigación empírica, la mayoría de los trabajos que estudian este tópico se basan en el enfoque econométrico lineal de Robert Barro, expuesto en su trabajo seminal de 1991. Barro (1991) utiliza datos de corte transversal para predecir la tasa de crecimiento per cápita de los países, intentando verificar las principales conclusiones de los modelos de crecimiento económico neoclásicos, fundamentalmente, la hipótesis de convergencia condicional que se deriva del modelo neoclásico de Solow-Swan ampliado por Mankiw, Romer y Weil (1992). Por esto último, los trabajos subsiguientes que adoptaron este enfoque, tanto a nivel de corte transversal o panel, fueron denominados alternativamente regresiones de crecimiento o regresiones de convergencia condicional. Alternativamente, como veremos en la Sección 2, existen modelos no lineales o con umbrales (Bick, 2010) ya sea en el marco de una regresión de convergencia condicional o modelos de crecimiento endógeno (Jones y Manuelli, 1995) que utilizan calibración de parámetros para predecir la tasa de variación del consumo o PBI real per cápita de equilibrio.

Brevemente, en el marco de un análisis de regresión múltiple, probar la hipótesis de convergencia condicional implica encontrar, en la ecuación que predice la tasa de crecimiento del PBI per cápita promedio para un período determinado, un signo negativo y significativo en el coeficiente que acompaña al nivel de PBI per cápita inicial. Debido a que un ejercicio econométrico que sólo utilizara dicho predictor estaría afectado por omisión de variables y endogeneidades de todo tipo, se procede a incorporar una serie de variables exógenas de control para explicar el efecto ceteris paribus en la tasa de crecimiento per cápita (dando cuenta de los diferentes estados estacionarios hacia los cuales convergería cada economía). Entre estas variables, suele incluirse la tasa de inflación de la economía. En general, los estudios econométricos que testean esta teoría 
han encontrado una asociación negativa entre la inflación y el crecimiento del PBI per cápita (Sala-i-Martin, 1997; Levine y Zervos, 1998; Barro, 2013).

En cuanto a los modelos no lineales y/o con umbrales, si los efectos negativos de la inflación en el crecimiento están asociados a factores como la incertidumbre, el acortamiento de los horizontes de planeamiento, la aleatoriedad de los precios relativos, etc., entonces el efecto marginal de la inflación no debería ser el mismo si el nivel es alto y explosivo (y, entonces, con mayor variabilidad) que si la inflación es baja, predecible y estacionaria. Siendo así, la efectividad de una reducción de la inflación de, por ejemplo, el 50\%, depende del nivel de partida: probablemente sea poco efectivo reducir la inflación de $2000 \%$ a $1000 \%$, y también lo sea reducirla del $2 \%$ al $1 \%$, pero puede tener un gran efecto reducirla del $20 \%$ al $10 \%$. Dicho con más rigor, en términos estadísticos: la magnitud de la correlación parcial entre inflación y crecimiento económico es condicional al nivel de inflación. Esto introduce dos efectos de interés. Por un lado, existen efectos de umbral: el efecto de la inflación en el crecimiento es significativo sólo a partir de cierto valor (o sólo en cierto intervalo, si también existe un umbral superior a partir del cual pierden significatividad). Por otro lado, el coeficiente de regresión es no lineal.

En cualquier caso, las regresiones del crecimiento pueden presentar una serie de problemas como sesgos de endogeneidad debido a variables omitidas, simultaneidad o errores de medición que no permitan una adecuada estimación del efecto de la inflación sobre el crecimiento de largo plazo y tampoco una predicción satisfactoria, en este contexto, del mismo, en particular en economías emergentes y en desarrollo donde la volatilidad de las variables macroeconómicas y su inestabilidad de largo plazo han sido recurrentes. Por lo tanto, el uso y aplicación de los estudios existentes sobre el tema podrían ser una guía imperfecta e incompleta para entender cómo interaccionan estas variables en economías emergentes o de frontera, dado que se obtendría una estimación imprecisa, afectada por el sesgo y la varianza del estimador, y predicciones con errores significativos y relativamente importantes en tamaño.

Se mencionó que la inflación es un fenómeno que tiene una relación compleja con el crecimiento económico, con diversos vasos comunicantes, y que las aproximaciones empíricas a dicha relación tienen algún tipo de debilidad asociado con endogeneidades diversas, o con la forma funcional impuesta (lineal o no lineal). Esta crítica metodológica habla de la validez interna de los modelos econométricos: hasta qué punto las conclusiones que se derivan de ellos pueden considerarse cuantitativamente exactas y pasibles de ser interpretadas como una relación causal. Sin embargo, no sólo interesa la validez interna, sino también la validez externa: hasta qué punto los resultados son generalizables. En la práctica, como señala Rodrik en el epígrafe de este trabajo, no importa sólo la exactitud cuantitativa o el grado de identificación causal (la validez interna) sino, principalmente, aprender a través del modelo algo que no era obvio y que enriquece el entendimiento que se tenía a priori del fenómeno (aspectos asociados con la validez externa). Es natural, por lo tanto, preguntarnos por la validez externa de los modelos. 
Una forma de evaluarla es someterlos a la crítica práctica: utilizarlos para intentar predecir información nueva, distinta a la utilizada para entrenarlos, y analizar qué tan bien predicen estos nuevos datos.

En este trabajo revisamos la literatura empírica sobre la relación entre inflación y crecimiento a largo plazo, intentando responder dos preguntas:

1. ¿Cuál es el error de predicción de los modelos (a la Barro y otros con umbrales y no linealidades) si se utilizan datos actuales para una muestra de países emergentes y en desarrollo?

2. ¿Cuáles son los principales problemas de estimación y predicción de dichos modelos y qué variantes se han adoptado o podrían adoptarse para mejorar tanto la validez interna como externa de los modelos?

El resto de este trabajo se organiza de la siguiente manera: en la sección 2, utilizaremos distintas versiones de las ecuaciones de convergencia condicional y de modelos con umbrales o no linealidades que incorporan la inflación como variable explicativa del crecimiento para realizar simulaciones, aplicándolas a un grupo de países calificados como mercados emergentes (siguiendo la clasificación de MSCI - Morgan Stanley Capital Investment-). Utilizaremos para ello, principalmente, datos provistos por el Banco Mundial, complementados con otras fuentes de datos cuando sea necesario. Estudiaremos la precisión de la predicción obtenida a través de una medida del error, la Raíz del Error Medio Cuadrático o Root Mean Squared Error (RMSE por su sigla en inglés). En la sección 3, realizaremos una revisión crítica de la literatura empírica sobre el tema, intentando identificar los principales problemas que podrían generar una estimación sesgada y/o inconsistente de la relación entre inflación y crecimiento económico. La sección 4 presenta las conclusiones y algunas recomendaciones.

\section{SIMULACIÓN PARA LOS MERCADOS EMERGENTES: LA VALIDEZ EXTERNA DE LAS REGRESIONES DEL CRECIMIENTO}

En esta sección comprobaremos hasta qué punto los resultados de un conjunto de investigaciones empíricas seleccionadas y representativas que estudian la relación entre inflación y crecimiento tienen poder predictivo y en qué medida. Técnicamente, esto implica detectar si los coeficientes estimados, a partir de datos para países y períodos concretos, generan predicciones más o menos precisas cuando se los expone a nuevos datos.

\subsection{Modelos SELECCIONADOS}

Existe una amplia variedad de investigaciones econométricas relativas a este tópico, desde modelos básicos estimados por mínimos cuadrados hasta modelos no lineales semi-paramétricos, pasando por una amplia gama de modelos lineales y no lineales. La estructura 
y las debilidades de estos distintos enfoques los discutiremos en la sección 3. A los fines de nuestro trabajo, adoptaremos una primera clasificación de esos modelos, dividiéndolos entre a) lineales (Barro (2013), Fischer (1993), Cukierman et al. (1993), Levine y Zervos (1998), Levine y Easterly (2001)), y/o b) no lineales y/o con umbrales, (Easterly y Bruno, 1998; Bick, 2010; Kremer, Bick y Nautz, 2011 y Baglan y Yoldas, 2014). Ciertamente, existen otros trabajos que abordan específicamente el caso de estudio de este trabajo (los mercados emergentes) como De Gregorio (1996), con datos hasta 1985, o Bittencourt (2012), para un puñado de países latinoamericanos y con una regresión algo simplista. La elección de estos modelos se atribuye a dos razones: a) que incluyen cortes transversales o paneles de un número representativo de países emergentes para un período amplio de tiempo, y b) que los trabajos hayan sido publicados en revistas con referato Q1 en el indexador Scimago. El siguiente cuadro resume las características de la muestra utilizada en cada uno (cantidad de países incluidos y el período de tiempo involucrado), y la cantidad de variables de control — distintas de la inflación — utilizadas en la ecuación seleccionada:

Cuadro 1. Cantidad de variables y detalle de la muestra, por modelo

\begin{tabular}{lcll}
\hline \multicolumn{1}{c}{ Trabajo } & $\begin{array}{c}\text { Cantidad de variables } \\
\text { explicativas }\end{array}$ & \multicolumn{1}{c}{ Países } & Período \\
\hline Barro (2013) & 15 & 100 & $1960-1990$ \\
Bick (2010) & 5 & 40 (Developing) & $1960-2004$ \\
Cukierman et al. (1993) & 6 & 60 (Industrial \& LCD) & $1960-1989$ \\
Easterly y Bruno (1998) & 6 & 31 (países con crisis) & $1961-1994$ \\
Fischer (1993) & 3 & Entre 22 y 94 (según ecuación) & $1960-1990$ \\
Kremer et al. (2011) & 7 & 124 & $1950-2004$ \\
Levine y Easterly (2001) & 6 & Entre 63 y 67 & $1960-1995$ \\
Levine y Zervos (1998) & 7 & 47 & $1976-1993$ \\
Baglan y Yoldas (2014) & 7 & 92 & $1975-2004$ \\
\hline
\end{tabular}

El gráfico 1 exhibe la estimación cuantitativa del efecto marginal de la inflación en el crecimiento de cada modelo ${ }^{2}$ :

2 El efecto marginal mostrado en los modelos no lineales se aproxima de la siguiente forma: en Bick (2010) y Kremer et al. (2011) se calcula, en un tramo representativo del dominio de la inflación, qué efecto promedio por punto de inflación representa el coeficiente asociado a un punto incremental de su logaritmo. En Baglan y Yoldas (2014) se presenta el asociado al punto crítico entre los dos umbrales que presentan, y en Easterly y Bruno (1998) el asociado a una situación discreta: las crisis inflacionarias. 
Gráfico 1. Efecto marginal de la inflación en el crecimiento

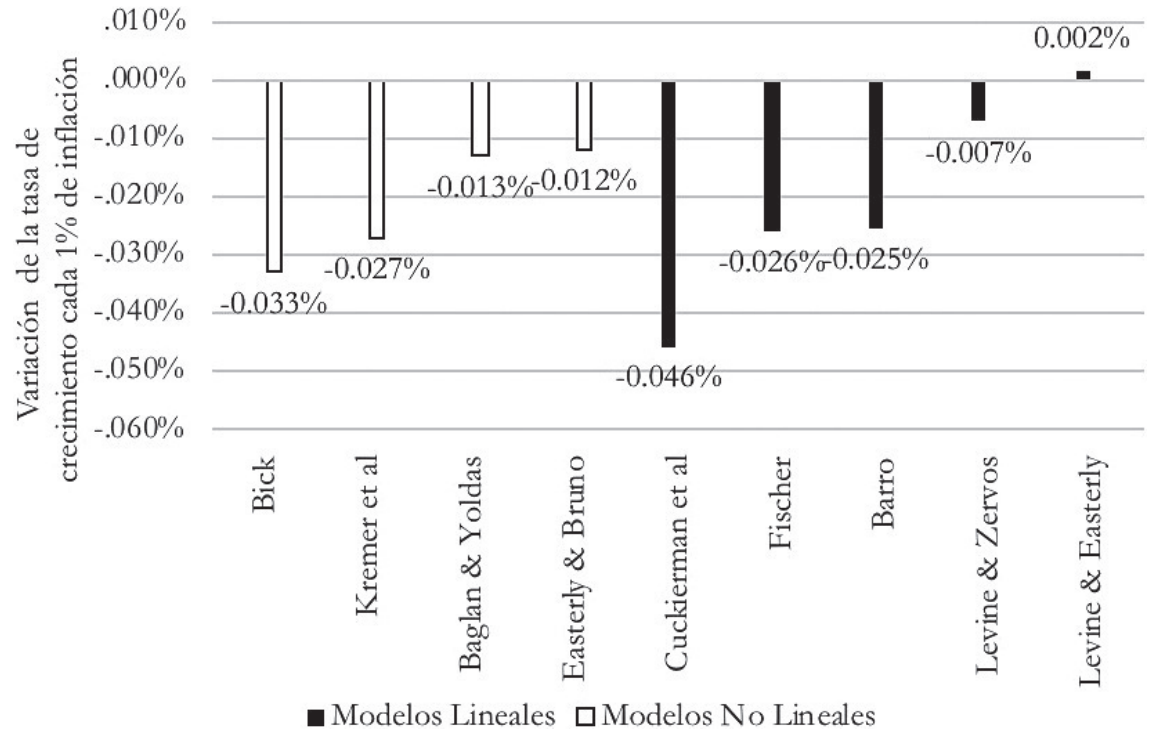

Elaboración propia en base al efecto marginal reportado por los autores: Baglan y Yoldas (2014) figura 6, Bick (2010) tabla 1, Easterly y Bruno (1998) tabla 10 regresión 1, Kremer et al. (2011) tabla 6, Barro (2013) tabla 2 ecuación 5, Fischer (1993) tabla 3 ecuación 11, Cukierman et al (1993) tabla 6 ecuación 4, Levine y Zervos (1998) tabla A1, y Levine y Easterly (2001) tabla 5.

Por ejemplo, Barro (2013) usando datos de corte transversal encuentra una relación monótonamente negativa, con un efecto marginal de la inflación en el crecimiento de aproximadamente 0,025. Esto implicaría que, e.g. una tasa de inflación $10 \%$ superior redundaría en una tasa de crecimiento económico $0,25 \%$ inferior, en promedio, ceteris paribus. Las simulaciones y predicciones utilizan todas las variables empleadas en los 9 modelos escogidos.

En esta Sección aplicaremos los coeficientes estimados en estos estudios a los datos de nuestra muestra de mercados emergentes, y analizaremos si las tasas de crecimiento predichas de esta forma se alinean razonablemente con los datos reales, lo que nos daría indicios de que los modelos tienen validez externa en el sentido de Rodrik. Las estadísticas que serán utilizadas para estas simulaciones se presentan en el Anexo, junto con un hipervínculo que permite la descarga de los datos.

\subsection{Problemas de medición de Variables}

Antes de mostrar los resultados y analizarlos, cabe mencionar que tratamos de replicar las variables utilizadas originalmente por los diversos autores en sus estudios. No obstante, existen algunas variables allí empleadas (e.g. índice de derechos políticos, índice de imperio de la ley, golpes y revoluciones, entre otras) cuya fuente no está disponible para este estudio. 
En esos casos, tratamos de ser lo más fiel posible a la elección de los autores, optando por variables que midan el mismo fenómeno con la mayor cercanía posible. Debe tenerse en cuenta que, aunque la regresión se haya estimado con una variable determinada, la aplicación de otra variable distinta será tan válida como la original siempre que esté medida en la misma escala, esté altamente correlacionada, tenga (en promedio) el mismo nivel y las diferencias entre la original y la nueva no sean sistemáticas sino aleatorias. Si bien pueden existir diferencias entre las variables utilizadas por los autores y las utilizadas en este trabajo, no hay motivos para sospechar priori un sesgo sistemático en ninguna de ellas. Las variables utilizadas son lo suficientemente fieles a las originales y, en caso de haber diferencias, el procedimiento realizado será equivalente a utilizar una variable con un error de medición aleatorio (es decir, no correlacionado con la variable original).

Afortunadamente, la mayoría de las variables para las cuales no existen series completas son secundarias en las estimaciones respectivas. Se trata de variables de control que inducen variabilidad y, cómo tales, deben tenerse en cuenta, pero no son las variables centrales de la regresión. Existe una excepción: el proxy de capital humano utilizado en Barro (2013), fundamental por su efecto en la convergencia. El mismo no está disponible ni es replicable (entre otras cosas, por estar centrado en torno a la media de la muestra utilizada por el autor), por lo que debió elaborarse un indicador lo más cercano posible al espíritu del trabajo en cuestión: una medida que combina el logaritmo de la cantidad de años de escolaridad secundaria en la población mayor de 25 ańos y el logaritmo de la esperanza de vida al nacer, ambas medidas tomadas para el año inicial de la muestra y estandarizadas.

Existen otros inconvenientes en la muestra que vale la pena mencionar. Primero, la medida de PBI per cápita elegida podría ser fuente de distorsiones: se encuentra valuada en dólares PPA de 2011, mientras que algunos de los modelos bajo estudio son muy anteriores y fueron estimados con un ańo base muy alejado de este último. Esto no solo afecta los residuos de la predicción (vía el parámetro de convergencia condicional que multiplica al PBI per cápita al inicio del período muestral) sino que distorsiona todas las medidas que impliquen centrar o estandarizar variables. Para remediar este problema, en los trabajos estimados durante los años 90 , se utilizará una medida alternativa con año base en 1996, para acercar la valuación a la fecha de estimación del modelo. Esto no elimina, pero reduce significativamente, dicho problema.

Segundo, la información primaria disponible para computar la prima del mercado de cambios paralelo (black market premium) es escasa y con muchos outliers (por ejemplo, Rusia a fines de la década de 1990), los cuales también serán suprimidos para no alterar la predicción. Lo mismo sucede con el período muestral: tal como se detalla en el cuadro 4 de la sección 3, antes de 1995 la muestra está afectada por valores que clasificamos como outliers. Los valores anómalos de la inflación absorben variabilidad que corresponde a otros fenómenos (shocks en los términos de intercambio en la década de 1970, crisis de deuda en la década de 1980 y principios de la de 1990). Si utilizáramos esos puntos para realizar simulaciones a partir de regresiones estimadas para amplios períodos y cuyo fin es pronosticar valores promedio, penalizaríamos indebidamente los modelos 
obteniendo un error de predicción muy elevado. Es por ello que, a fines de la predicción, utilizaremos las observaciones desde 1995 en adelante. No sólo porque es de mayor interés (dado que es información más actual), sino para dejar fuera de la simulación los períodos de inestabilidad que contienen la casi totalidad de los outliers.

Si bien remover valores anómalos no siempre es una práctica recomendada, tratar de esta forma los outliers está justificado a priori en el contexto de una simulación: la idea de realizar una simulación a partir de las ecuaciones estimadas es entender si predicen correctamente la evolución normal de una economía. Eliminar observaciones anómalas permite conservar el efecto de las distintas variables sobre aquellas coyunturas normales sin generar grandes desvíos que lleven a conclusiones distorsionadas sobre la utilidad del estudio en cuestión.

\subsection{Resultados DE PREDiCCIÓN}

\subsubsection{Primeros resultados: Barro (2013) y Fischer (1993).}

Teniendo presente los ajustes mencionados a las variables, y comenzando con el trabajo de Barro (2013) para predecir el crecimiento de los mercados emergentes en el período 1995-2017, se obtiene el siguiente resultado:

Gráfico 2. Simulación de Barro (2013). Valores observados vs. predichos, 1995-2016

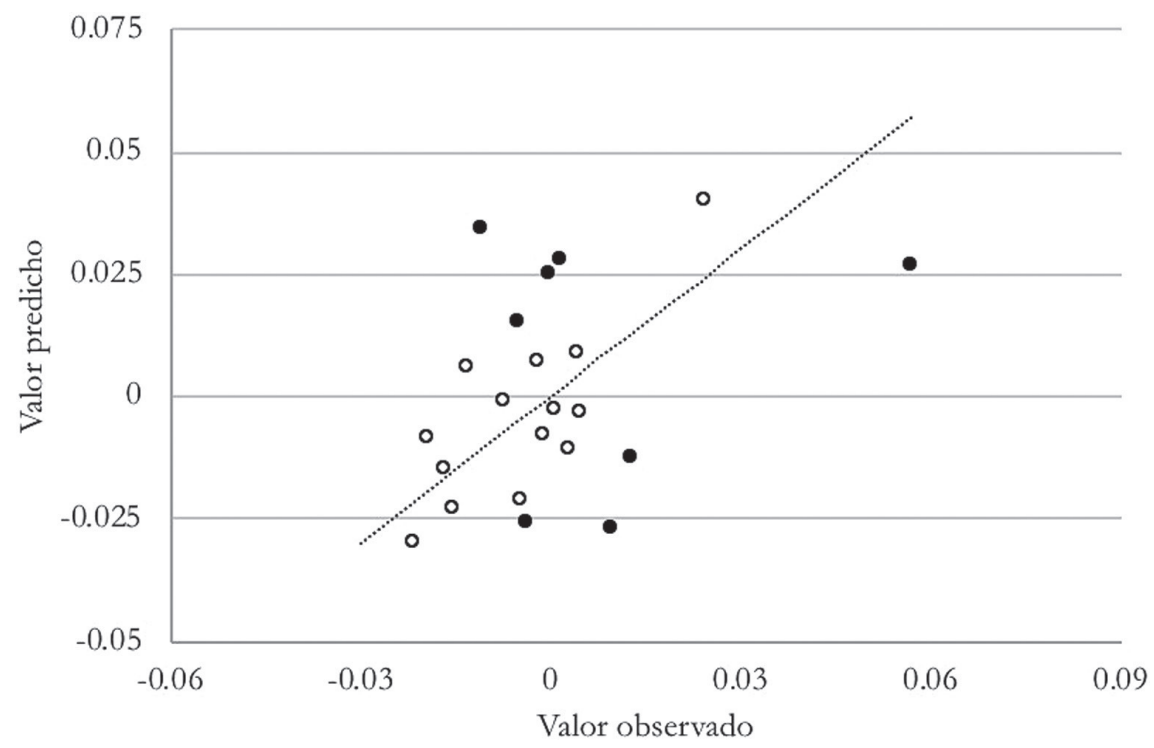

Elaboración propia. Valor observado (eje de abscisas) es el promedio del crecimiento centrado en la media del PBI per cápita para cada país de la muestra MSCI (excluidos Emiratos Árabes Unidos y Qatar), en el período 1995-2016. Valor predicho (eje de ordenadas) es el resultado de aplicar a los coeficientes de Barro (2013), tabla 2, ecuación 5 los valores correspondientes de cada variable para dichos países en el mismo período (excluyendo la interacción capital humano-PBI inicial). La línea punteada representa la recta de 45 grados, donde ambos valores son idénticos. Los puntos coloreados en negro son aquellos que presentan un error de predicción superior a 0.02 , representando las predicciones más imprecisas. 
Las regresiones de Barro tienen un intercepto que el autor no reporta, por lo que la simulación debió ser realizada centrando las variables en torno a la media para poder aplicar los coeficientes y obtener una estimación precisa sin necesidad de contar con dicho dato. En el gráfico 2, la distancia vertical entre el punto y la recta representa el error de predicción (sobre la recta de 45 grados, el valor predicho coincide con el valor real). Como se observa en los puntos negros del gráfico, la predicción es imprecisa para una importante cantidad de casos. ¿Cómo obtener una medida resumen de estas distancias? Uno de los estadísticos más populares a este fin es la raíz del error cuadrático medio $^{3}$ (RMSE, Root Mean Squared Error). Intuitivamente, esta medida puede interpretarse como el desvío estándar de la predicción, en el sentido de que es la raíz cuadrada del promedio muestral de las diferencias cuadráticas entre el valor observado y el valor predicho:

$$
R M S E=\sqrt{\frac{1}{N} \sum_{i=1}^{N}\left(Y_{i}-\hat{Y}_{i}\right)^{2}}
$$

Para esta simulación, el RMSE obtenido es de 0.137. Esta medida está expresada en las mismas unidades que la variable predicha, por lo que esta magnitud puede interpretarse como un desvío promedio de $13,7 \%$ en la tasa de crecimiento (centrada) predicha. Es un valor muy elevado y sin sentido económico. ¿A qué se debe tan baja performance? Barro incorpora una interacción entre el PBI per cápita inicial y el stock de capital humano inicial. Si se ignora dicha variable (siendo esta la simulación que se muestra en el gráfico 1), el RMSE baja a 0,019, un error aún considerable pero tolerable. Si bien eliminar dicho término no es del todo correcto porque capta un efecto de primer orden (la convergencia condicional) que acabaría formando parte del error, vale la pena mencionar tres argumentos que pueden explicar esta diferencia.

Primero, Barro no reporta a qué precios está medido el PBI per cápita que utiliza. Sin embargo, su estimación fue realizada en el año 1995, por lo que puede asegurarse que era una valuación distinta a la utilizada en este trabajo (en este caso, dólares PPA constantes de 1996). Por lo tanto, se trata de variables de una magnitud diferente. Segundo, si bien el índice de capital humano utilizado replica cualitativamente al de Barro, y aunque el mismo fue reescalado para que su valor absoluto esté contenido entre -1 y 1 , aún podría ser de un orden de magnitud distinto al original, lo que impactaría en la predicción. Finalmente, es llamativa la magnitud del coeficiente asociado a la interacción entre PBI per cápita y capital humano: su valor es muy superior al coeficiente individual de cada una de las variables que forman parte de ella. Por ejemplo, es 20 veces

3 Sobre la definición e interpretación de esta métrica para evaluar la performance de modelos predictivos, véase, por ejemplo, Barreto y Howland (2006, pp. 114-122). 
superior al coeficiente asociado al PBI inicial y 10 veces superior al de esperanza de vida. Si en lugar de un coeficiente de $-0,45$ (el reportado por Barro) se utilizara uno de -0,045, el RMSE caería a 0,022. Descartando que exista un error en el reporte de Barro, podría suceder que el término en cuestión esté medido en una escala diferente.

El modelo estimado por Barro estudiado en los párrafos previos presenta una particularidad: utiliza 17 covariables para estimar la tasa de crecimiento per cápita, cuando el resto de los autores utilizan un promedio de 8 . El modelo de Barro es el que menos simplicidad presenta; en términos estadísticos, es el que menos satisface la propiedad de parsimonia. Existe un trade-off entre parsimonia y bondad del ajuste (tal como medida, por ejemplo, por el $R^{2}$ ajustado): cuanto menos parsimonioso es un modelo (es decir, cuantos más regresores utiliza) mejor ajusta a los datos.

Un modelo parsimonioso presenta un beneficio: aun cuando tenga costo en términos de validez interna (lo cual no sucedería si las variables adicionales incorporadas en un modelo más complejo resultasen irrelevantes en la ecuación estructural), podría tener mayor validez externa: la simplicidad implica que es más probable una generalización exitosa. Esto sucede porque modelos complejos que incorporan variables de creciente grado de irrelevancia conducen a situaciones conocidas como overfitting: los parámetros estimados absorben el ruido aleatorio presente en los datos de la muestra y, si bien se ajusta muy bien los datos con los cuales se están estimando los parámetros del modelo, éste predice mal información no observada al momento de estimarlos.

Veamos cómo impacta esta discusión en nuestro estudio: el modelo con mayor parsimonia de los considerados en este trabajo es el de Fischer (1993), basado en el enfoque de la contabilidad del crecimiento. Fischer utiliza cuatro variables: inflación, resultado financiero del sector público, evolución de los términos de intercambio y el diferencial de tasa de cambio en el mercado paralelo y el oficial (black market premium) — como proxy de las distorsiones en los mercados - para estimar la tasa de crecimiento, y obtiene un coeficiente asociado a la inflación muy similar al obtenido por Barro: -0,026. En términos de validez interna, para el tópico de interés en este trabajo (no así para el modelo en general), ambos modelos son equivalentes: identifican un efecto de la inflación en el crecimiento de magnitud similar.

En términos de validez externa, al aplicar los coeficientes estimados por Fischer a nuestra base de datos de mercados emergentes, se obtiene un RMSE de 0,021. Graficando los valores estimados frente a los reales, se observa lo siguiente: 
Gráfico 3. Simulación de Fischer (1993). Valores observados vs. predichos, 1995-2016

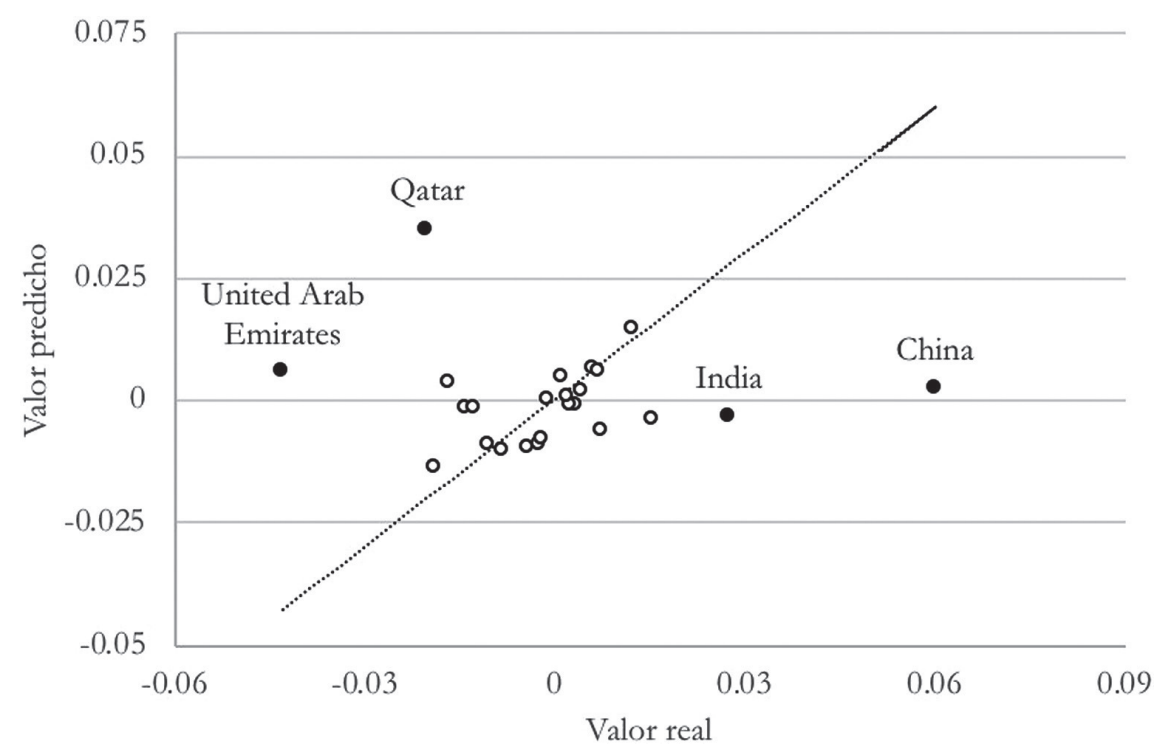

Elaboración propia. Valor observado (eje de abscisas) es el promedio del crecimiento centrado en la media del PBI per cápita para cada país de la muestra MSCI, en el período 1995-2016. Valor predicho (eje de ordenadas) es el resultado de aplicar a los coeficientes de Fischer (1993) tabla 3, ecuación 11, los valores correspondientes de cada variable para dichos países en el mismo período (excluyendo la interacción capital humano-PBI inicial). La línea punteada representa la recta de 45 grados, donde ambos valores son idénticos. Los puntos coloreados en negro son aquellos que presentan un error de predicción superior a 0,02 , representando las predicciones más imprecisas.

La mayor parte de este desvío es explicado por cuatro países: Qatar, Emiratos Árabes Unidos, China e India. Esto probablemente represente un costo de la simplicidad del modelo: por ejemplo, los dos últimos países han atravesado un proceso de cambio estructural notable que no es captado por las variables utilizadas. $\mathrm{Al}$ remover estas cuatro observaciones, se obtiene un RMSE corregido de 0,0085. Recuérdese que, al corregir las anomalías en el caso de Barro (2013), el valor obtenido fue de 0,019, más del doble que el de Fischer (1993). Salvo en el caso de India, los casos peor predichos en Barro (2013) son distintos a los de Fischer (1993).

En resumen, la estimación de Fischer (1993) presenta una serie de ventajas frente a la de Barro (2013): es más simple, predice mejor ante datos nuevos que no formaron parte de la muestra con la cual se estimó (tiene mayor validez externa) y, aunque predice mal países en un rápido proceso de convergencia o cambio estructural (dado que omite variables relevantes; por ejemplo, no incluye ninguna variable para controlar el efecto de convergencia condicional), obtiene un coeficiente para la inflación virtualmente idéntico al de Barro, lo que conduciría a conclusiones y recomendaciones de política equivalentes en ambos modelos. 


\subsubsection{Resultados generales para todos los modelos}

A continuación, presentamos el poder predictivo de las dos variedades de modelos escogidos como se explicó en la Sección 1, i.e. lineales y no lineales y/o con umbrales.

Existen dos países cuyo elevado PBI per cápita tiene un impacto enorme en las tasas predichas a través de los coeficientes que explican la convergencia condicional: Qatar y Emiratos Árabes. Para evitar penalizar excesivamente el RMSE de los modelos por estos dos casos, se removerán de la muestra. Con esta salvedad, entonces, al aplicar a las ecuaciones de cada modelo los datos de los mercados emergentes en el período 1995-2016 se obtiene el siguiente RMSE expresado como proporción de la tasa de crecimiento promedio del período (PRMSE, Porcentual Mean Squared Error):

Gráfico 4. Error de predicción por modelo, en porcentaje. 1995-2016

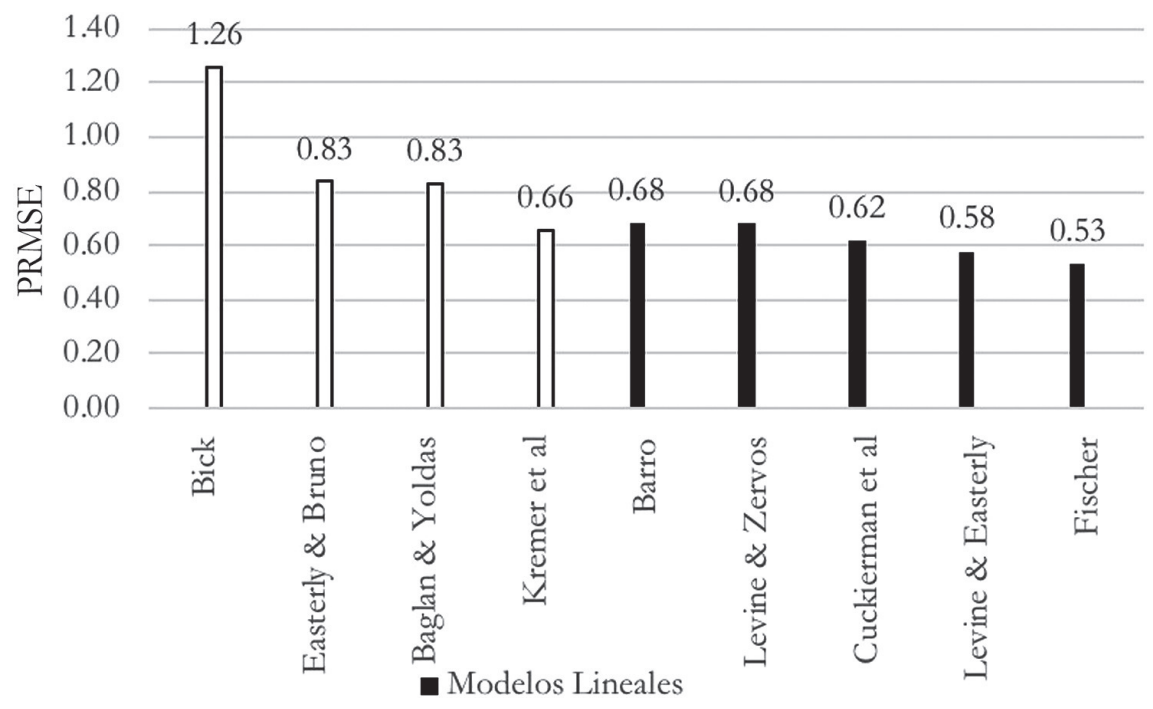

Elaboración propia. PRMSE es el RMSE dividido por la tasa promedio de crecimiento per cápita en el período 1995-2016 para los mercados emergentes. RMSE obtenido como el promedio de la suma de los residuos cuadráticos de las predicciones de cada modelo para los países de la muestra.

Este gráfico revela algunos hechos interesantes. Primero, el modelo de Fischer es el que mejor predice en el marco de este ejercicio (de forma relativa a los otros modelos), seguido por Cukierman (1993) en el tercer lugar, y dos que incorporan variables de desarrollo financiero en el segundo y cuarto lugar: Levine y Easterly (2001) y Levine y Zervos (1998). No obstante, todos los modelos presentan desvíos elevados, aún después de la remoción de anomalías, producto de los factores señalados previamente: fundamentalmente, que se trata de estimaciones realizadas para otros períodos y para países cuya estructura económica es diferente a la de los mercados emergentes en análisis. Segundo, los modelos lineales tienden a predecir con mayor precisión relativa que los 
no lineales o con umbrales, que por su parte sobreestiman el verdadero impacto de la inflación con mayor frecuencia.

Para avanzar en el análisis, podemos descomponer el RMSE de cada modelo por país. En el gráfico 5, donde el color negro indica una sobreestimación, y el color blanco una subestimación del crecimiento, se observa que en algunos casos (Bick, 2010; Kremer et al, 2011; Baglan y Yoldas, 2014) el error se debe a un sesgo sistemático a sobre o subestimar la tasa de crecimiento de los países. En otros casos (Fischer, 1993; Levine y Easterly, 2001; Levine y Zervos, 1998) no hay un patrón sistemático y el error se debe a algunas predicciones específicas con errores relativos mayores:

Gráfico 5. Error de predicción por país, 1995-2016
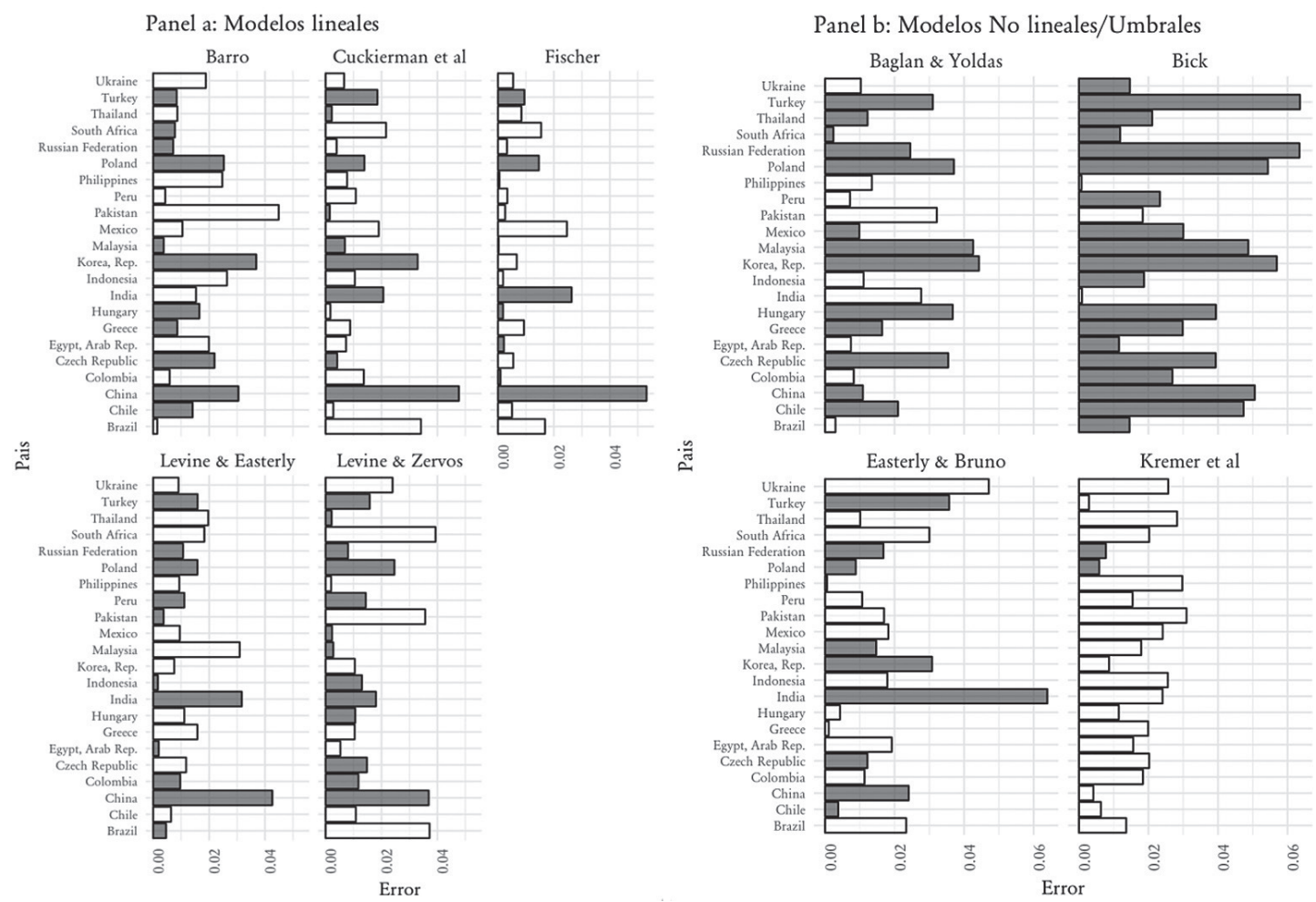

Elaboración propia. Diferencia entre la tasa observada de crecimiento per cápita promedio para 1995-2016 y la predicha por cada modelo. El color blanco indica una subestimación (la tasa predicha es inferior a la observada). El color negro indica una sobreestimación (la tasa observada fue inferior a la predicha).

Dando un paso adicional, podemos analizar la correlación entre los vectores de predicciones por país generados por cada modelo. Si plasmamos esta información en una matriz de correlaciones, obtenemos el siguiente resultado: 
Cuadro 2. Correlación entre predicciones de modelos, 1995-2016

\begin{tabular}{|c|c|c|c|c|c|c|c|c|}
\hline & $\begin{array}{c}\text { Levine } \\
\text { y Easterly }\end{array}$ & $\begin{array}{c}\text { Cuckierman } \\
\text { et al. }\end{array}$ & $\begin{array}{l}\text { Easterly } \\
\text { y Bruno }\end{array}$ & $\begin{array}{c}\text { Levine } \\
\text { y Zervos }\end{array}$ & $\begin{array}{l}\text { Kremer } \\
\text { et al. }\end{array}$ & Barro & $\begin{array}{c}\text { Baglan } \\
\text { y Yoldas }\end{array}$ & Bick \\
\hline $\begin{array}{l}\text { Baglan } \\
\text { y Yoldas }\end{array}$ & & & & & & & & 0,91 \\
\hline Barro & & & & & & & 0,87 & 0,82 \\
\hline $\begin{array}{l}\text { Kremer } \\
\text { et al. }\end{array}$ & & & & & & 0,70 & 0,75 & 0,76 \\
\hline $\begin{array}{c}\text { Levine } \\
\text { y Zervos }\end{array}$ & & & & & 0,33 & 0,21 & 0,4 & 0,49 \\
\hline $\begin{array}{l}\text { Easterly } \\
\text { y Bruno }\end{array}$ & & & & 0,25 & 0,21 & 0,25 & 0,34 & 0,33 \\
\hline $\begin{array}{c}\text { Cukierman } \\
\text { et al. }\end{array}$ & & & 0,46 & 0,02 & 0,19 & 0,33 & 0,49 & 0,38 \\
\hline $\begin{array}{l}\text { Levine } \\
\text { y Easterly }\end{array}$ & & $-0,25$ & 0,01 & 0,12 & 0,39 & $-0,09$ & $-0,22$ & $-0,05$ \\
\hline Fischer & 0,47 & 0,01 & $-0,02$ & 0,17 & 0,26 & $-0,09$ & $-0,04$ & $-0,07$ \\
\hline
\end{tabular}

Elaboración propia. Coeficiente de correlación entre las predicciones generadas por cada modelo para la tasa media de crecimiento de cada país en el período 1995-2016.

Esta matriz muestra que, en buena medida, las predicciones generadas tienen una correlación positiva moderada/alta, lo que implicaría una redundancia: la información que otorgan los distintos modelos es similar. Recuerde el lector que el período de predicción es en todos los casos 1995-2016, pero el período de estimación de los parámetros de cada modelo de regresión no es necesariamente idéntico (ver Cuadro 1 arriba).

A primera vista, dos casos son la excepción al respecto: el modelo de Fischer (1993) y el de Levine y Easterly (2001). La correlación baja/negativa entre estos modelos y los demás implica que ambos revelan patrones en los datos que los demás no aprovechan, omiten o explotan de manera diferente. Asimismo, tal como muestra el gráfico 5 de RMSE, ambos están entre los modelos con menor error de predicción. El modelo de Fischer (1993), tal como mencionamos previamente, es el más simple, lo cual parece darle ventaja para estilizar los hechos bajo estudio (obteniendo mayor validez externa). El modelo de Levine y Easterly (2001) es uno de los que introduce al desarrollo financiero, por lo que incorpora información que los demás modelos pasan por alto. El otro modelo que tiene en cuenta esto, el de Levine y Zervos (1998), si bien tiene una correlación positiva con casi todos los modelos, esta es moderada/baja, lo que apoyaría estas conclusiones. En un orden de magnitud diferente, los modelos que incorporan no linealidades también presentan una correlación moderada con el subsegmento de los modelos 
lineales, (excepto con el caso de Barro donde la correlación es muy alta ${ }^{4}$ ). También aquí sucede que, al permitir no-linealidades en la relación entre inflación y crecimiento, incorporan información que los demás modelos omiten.

Resumiendo, encontramos hasta aquí que los modelos simples (parsimoniosos) son importantes para potenciar la capacidad de generalización hacia nueva información, y que, por otro lado, la variable desarrollo financiero y la no-linealidad en la relación entre inflación y crecimiento son relevantes para detectar patrones en los datos que los enfoques tradicionales omiten. Finalmente, un llamado a tomar con cautela la validez externa de estos modelos: el error de predicción obtenido no es pequeño. Atribuimos esto, principalmente, a que fueron estimados en otros períodos y con países estructuralmente distintos a los utilizados en el presente estudio. Esto no quiere decir que los mercados emergentes no formaban parte de las respectivas muestras utilizadas, sino que la composición muestral era diferente: los países calificados como mercados emergentes por MSCI estaban subrepresentados en comparación con el estudio actual, donde forman el $100 \%$ de la muestra. Estos países no son calificados de esta forma de manera aleatoria, sino por cumplir una serie de requisitos. Esto es: parte del RMSE elevado podría ser explicado por una suerte de sesgo de selección en la muestra a la cual estamos aplicando los modelos.

Si las diferencias estructurales explican los errores de predicción, entonces una nueva estimación basada en estos países lograría una modelización más precisa de su crecimiento económico y una identificación más exacta del vínculo causal entre inflación y crecimiento presente en dichas economías 5 . Pero, además, la fuente de este problema puede estar en la estructura misma de los modelos. En este punto, retomamos lo expuesto en la introducción: uno de los predictores utilizados, la inflación, presenta problemas cuando se lo utiliza como predictor de la tasa de crecimiento económico. Estudiar esta problemática (que implica virar el enfoque hacia la validez interna de los modelos) será el objetivo de la próxima sección.

4 Es llamativo que los modelos que más se alejan del enfoque de regresiones del crecimiento (Bick, 2010; Baglan y Yoldas, 2014; y Kremer et al., 2011) generen predicciones tan alineadas con las del padre de dicho enfoque.

5 Una forma de buscar pistas respecto a esta hipótesis de la diferencia estructural sería estudiar modelos en los cuales se estime la relación entre inflación y crecimiento con muestras compuestas sólo con mercados emergentes (o, como aproximación más cercana, con países en vías de desarrollo, de los cuales estos mercados son un subconjunto). La evidencia en este sentido es mixta. Bittencourt (2012), en un panel con datos de 1970-2007 para países latinoamericanos que atravesaron crisis hiperinflacionarias, encuentra un coeficiente de entre $-0,0164$ y -0,0216 (que llega a un -0,04 cuando estima por variables instrumentales), en línea con los modelos bajo análisis. En cambio, De Gregorio (1996) detecta coeficientes ligeramente diferentes para el mundo en desarrollo durante el período 1960-1985 (-0,071 para países en desarrollo, contra - $-0,057$ en todos los países). De estos ejemplos pueden deducirse dos cosas: Primero, independientemente de la magnitud del efecto marginal, las estimaciones coinciden en el signo negativo. Segundo, en términos cuantitativos, no hay evidencia suficiente para asegurar que el efecto marginal de la inflación en el crecimiento sea de una magnitud estructuralmente diferente en países en vías de desarrollo. 
Previamente, debe realizarse un control de robustez de los resultados obtenidos. El período muestral utilizado para las simulaciones, tal como se mencionó anteriormente, es 1995-2016. Existen dos razones que justifican reformular los cálculos para una muestra acotada al período 2005-2016. Primero, si bien, por razones de comparabilidad, se utilizó la misma muestra para todos los modelos, tres de ellos (Baglan y Yoldas, 2014; Bick, 2010 y Kremer et al., 2011)) fueron estimados en los años 2000 y sus muestras incluían parte de los años y países considerados en este trabajo, por lo que los de nuestra muestra no son totalmente datos nuevos para ellos. Segundo, durante los últimos años (principalmente desde mediados de la década de 2000, ver gráfico 7 más adelante), la inflación se ha mantenido en niveles bajos, por lo que los resultados podrían estar sesgados debido a la aplicación de los coeficientes a un período de inflación en descenso. Esto último sucedería porque, si la relación estructural subyacente entre la inflación y el crecimiento es no lineal o con umbrales, los modelos que asumen una relación lineal podrían estar sobreestimando el impacto de una inflación en descenso, o esta podría estar inactivando los umbrales (al posicionarse por debajo de ellos).

En el cuadro 2 se exponen los resultados de este ejercicio de robustez. Al volver a simular con esta muestra acotada, se genera un reordenamiento intragrupo en los modelos lineales, pero los modelos no lineales siguen presentando, en promedio, los mayores errores de predicción. El modelo de Fischer (1993) deja de ser aquel con menor PRMSE, lugar que pasa a ocupar el modelo de Levine y Zervos (1998), uno de los que incorpora al desarrollo financiero entre las variables explicativas. ¿̇Alteran estos resultados nuestras conclusiones? Vale la pena resaltar algunos puntos al respecto:

1) Como ya se mencionó, los modelos no lineales siguen siendo aquellos con un mayor PRMSE. Sin embargo, en términos generales, las diferencias en PRMSE de mayor valor absoluto entre ambas simulaciones son cuatro: un modelo lineal cuya performance mejora (Bick, 2010), y tres modelos no lineales, de los cuales dos empeoran su resultado, Levine y Easterly (2001) y Fischer (1993), siendo el de Levine y Zervos (1998) el único modelo lineal con una mejora considerable. Aunque en términos absolutos los modelos no lineales o con umbrales continúan siendo los de mayor error de predicción, en términos diferenciales son los más beneficiados por el cambio muestral. Esto podría ser evidencia a favor de lo mencionado anteriormente: que la relación estructural subyacente sea no lineal, y por lo tanto utilizar el período muestral con menor inflación promedio beneficia a los modelos que incorporan esto en su especificación.

2) Por otro lado, si bien Fischer (1993) deja de ser el modelo con mejor performance, esto no es un argumento en contra del punto hecho a favor de los modelos parsimoniosos, sino que puede ser señal de que dicho modelo ignora variables relevantes (se señaló que no incorpora variables relacionadas con la convergencia condicional, como el PBI per cápita inicial o el capital humano). 
3) En cuanto al rol del desarrollo financiero, se debilita la performance del modelo de Levine y Easterly (2001), pero mejora la de Levine y Zervos (1998), por lo que no emerge de este ejercicio evidencia en contra de lo expuesto. En la siguiente sección se analizará en detalle la diferencia entre ambos modelos y cómo impacta esto en las estimaciones.

Cuadro 2. Error de predicción por modelo, muestra acotada (2005-2016) vs. muestra completa (1995-2016)

\begin{tabular}{lccc}
\hline \multirow{2}{*}{ Modelo } & $(1)$ & $(2)$ & $(3)$ \\
\cline { 2 - 4 } & $\begin{array}{c}\text { PRMSE muestra } \\
\text { acotada }\end{array}$ & $\begin{array}{c}\text { PRMSE muestra } \\
\text { completa }\end{array}$ & $\begin{array}{c}\text { Diferencia } \\
(1)-(2)\end{array}$ \\
\hline Bick (2010) & 1,10 & 1,26 & $-0,16$ \\
Easterly y Bruno (1998) & 0,86 & 0,83 & 0,03 \\
Baglan y Yoldas (2014) & 0,79 & 0,83 & $-0,04$ \\
Kremer et al. (2011) & 0,72 & 0,66 & 0,06 \\
Levine y Easterly (2001) & 0,72 & 0,58 & 0,14 \\
Fischer (1993) & 0,65 & 0,53 & 0,12 \\
Barro (2013) & 0,62 & 0,68 & $-0,04$ \\
Cukierman et al. (1993) & 0,62 & 0,62 & 0 \\
Levine y Zervos (1998) & 0,59 & 0,68 & $-0,09$ \\
\hline
\end{tabular}

\section{LA ESTIMACIÓN EMPÍRICA DEL EFECTO DE LA INFLACIÓN EN EL CRECIMIENTO ECONÓMICO DE LARGO PLAZO Y LA VALIDEZ INTERNA DE LOS MODELOS ECONOMÉTRICOS}

Discutir la validez interna de las regresiones del crecimiento para identificar el efecto de la inflación refiere a discutir si se cumplen las condiciones para que el coeficiente estimado pueda ser identificado con un efecto causal. Esto es problemático cuando, por ejemplo, se registra simultaneidad, omisión de variables o errores de especificación. En esta sección, analizaremos en qué medida estos problemas están presentes en los estudios que investigan la relación entre inflación y crecimiento.

i. Endogeneidad en las ecuaciones de convergencia condicional: superneutralidad del dinero y correlación entre inflación y crecimiento

Kocherlakota (1996) menciona que existe una correlación negativa entre inflación y crecimiento, aun cuando el dinero sea superneutral, por medio de la condición de equilibrio del mercado de dinero. Esta condición, en una versión simplificada y asumiendo 
que la demanda de dinero es idéntica al producto nominal (esto es: solo existe demanda por motivo transaccional), nos dice que:

$$
M_{t}^{s}=M_{t}^{d}=Y_{t}=y_{t} P_{t} \rightarrow \frac{M_{t}^{s}}{y_{t}}=P_{t}
$$

Donde $M_{t}^{s}$ y $M_{t}^{d}$ son, respectivamente, la oferta y la demanda de dinero, $y_{t}$ es el producto en términos reales, $Y_{t}$ es el producto en términos nominales, y $P_{t}$ es el nivel de precios. Nótese que esta condición es susceptible de ser derivada de la ecuación cuantitativa del dinero (que, en cuanto identidad contable, se cumple en todo momento), provisto que nuestro supuesto sobre la demanda de dinero implica una velocidad de circulación igual a 1. A partir de esta condición, para que el mercado de dinero esté en equilibrio inter temporalmente, debe cumplirse que:

$$
\begin{aligned}
& \ln \left(M_{t}^{s}\right)-\ln \left(y_{t}\right)=\ln \left(P_{t}\right) \\
& \frac{\partial \ln \left(M_{t}^{s}\right)}{\partial t}-\frac{\partial \ln \left(y_{t}\right)}{\partial t}=\frac{\partial \ln \left(P_{t}\right)}{\partial t} \rightarrow m_{t}-g_{t}=\pi_{t}
\end{aligned}
$$

Donde $m_{t}$ es la tasa de crecimiento de la oferta monetaria, $g_{t}$ la tasa de crecimiento del producto, y $\pi_{t}$ la inflación del período. Entonces, el equilibrio del mercado de dinero implica que los precios crezcan a una tasa igual a la brecha entre el crecimiento de la oferta de dinero y el crecimiento del producto. Obsérvese que, si los componentes de la demanda de dinero distintos al transaccional son estables en términos reales (lo que significa que su tasa de crecimiento oscila en torno a 0), esta identidad es robusta al relajamiento de los supuestos sobre esta variable.

¿Qué nos dice esta condición de equilibrio sobre la correlación entre la tasa de crecimiento del producto y la tasa de variación del nivel general de precios? Como primer paso, consideremos la ecuación estructural que explica la esperanza condicional de la tasa de crecimiento:

$$
E\left(g_{t} \mid \pi_{t}, X_{t}\right)=\beta_{0}+\beta_{\pi} \pi_{t}+\sum_{1}^{p} B_{p} X_{p, t}
$$

Donde el término de la sumatoria representa el efecto de todos los predictores distintos de la inflación que explican la tasa de crecimiento, como vimos en la sección 2 . El efecto marginal de la inflación en esta ecuación depende del impacto que tenga el componente de dicha variable que induce variabilidad exógena en el crecimiento (es decir, el componente libre de endogeneidad). Este elemento es el residuo que queda luego de proyectar linealmente a la inflación sobre el resto de las variables $\left(X_{1}, \ldots, X_{p}\right)$, tal que: 


$$
\begin{aligned}
& \hat{\pi}_{t}=\alpha_{0}+\sum_{p=1}^{P} \alpha_{p} X_{p} \\
& \tilde{\pi}_{t}=\pi_{t}-\hat{\pi}_{t}
\end{aligned}
$$

Lo que esto nos dice, en términos intuitivos, es que el efecto marginal de la inflación en el crecimiento depende del efecto que tenga la parte de la inflación que no es explicada por el movimiento de las demás variables involucradas.

Entonces, si se cumple el supuesto de superneutralidad del dinero (Sidrauski, 1967), la inflación no afecta al crecimiento económico y el efecto marginal de este componente exógeno debería ser nulo en la ecuación estructural:

$$
B_{\pi}=\frac{\operatorname{cov}\left(\tilde{\pi}_{t}, g_{t}\right)}{\operatorname{var}\left(\tilde{\pi}_{t}\right)}=0
$$

Ahora bien ¿Cuál es la correlación total que observa el analista? Si se estimara la tasa de crecimiento de la economía respecto a la tasa de inflación mediante el método de los mínimos cuadrados ordinarios, se obtendría un coeficiente del tipo:

$$
\hat{\beta}_{\pi}^{o l s}=\frac{\operatorname{cov}\left(\pi_{t}, g_{t}\right)}{\operatorname{var}\left(\pi_{t}\right)}
$$

Reemplazando la tasa de inflación por su expresión en la identidad contable derivada previamente en la ecuación 3 y agrupando términos, se llega al siguiente coeficiente mínimo-cuadrático en la regresión lineal que explica la tasa de crecimiento económico a través de la tasa de inflación:

$$
\hat{\beta}_{\pi}^{\text {ols }}=\frac{\operatorname{cov}\left(m_{t}-g_{t}, g_{t}\right)}{\operatorname{Var}\left(m_{t}-g_{t}\right)}=\frac{\overbrace{E\left(g_{t} m_{t}\right)-E g_{t} E m_{t}}^{\operatorname{cov}\left(g_{t}, m_{t}\right)}-\overbrace{\left[E\left(g_{t}^{2}\right)-\left(E g_{t}\right)^{2}\right]}^{\operatorname{var}\left(g_{t}\right)}}{\operatorname{var}\left(m_{t}\right)+\operatorname{var}\left(g_{t}\right)-2 \operatorname{cov}\left(g_{t}, m_{t}\right)}
$$

Ahora bien, el supuesto de superneutralidad del dinero implicaría que la tasa de crecimiento del producto y la tasa de variación de la oferta monetaria sean eventos estadísticamente independientes y, por lo tanto, su covarianza debe ser nula. Por lo tanto:

$$
\operatorname{cov}\left(g_{t}, m_{t}\right)=0 \rightarrow \hat{\beta}_{\pi_{t}}^{o l s}=-\frac{\operatorname{var}\left(g_{t}\right)}{\operatorname{var}\left(m_{t}\right)+\operatorname{var}\left(g_{t}\right)}
$$

Donde el cociente es la suma de dos términos positivos (la varianza de una variable aleatoria es siempre superior a 0 ) y, por lo tanto, el efecto marginal estimado de la inflación sobre la tasa de crecimiento será negativo, aun cuando no exista una relación sistemática entre el producto y el nivel de precios. 
En resumen: aunque la verdadera relación entre la inflación y la tasa de crecimiento es nula si el dinero es superneutral, a raíz de un problema de simultaneidad es posible observar una correlación negativa entre ambas variables, porque el coeficiente estimado de la inflación podría estar capturando variabilidad endógena, inducida a través del efecto de otras variables.

¿Qué significa esto para las regresiones de convergencia condicional lineales o para los modelos con umbrales y/o no linealidades? Significa que, si se utiliza una estrategia de identificación inadecuada o incompleta, tal que la especificación del modelo no capture el efecto de las demás covariables sobre el crecimiento el producto, se estimará un coeficiente para la inflación que tendrá problemas de endogeneidad. Las dos formas extendidas con las cuales la literatura mitiga este problema es a través de regresiones por controles (agregando variables para capturar la endogeneidad y poder aislar su efecto ceteris paribus) y regresiones por el método de variables instrumentales o de momentos generalizados- GMM, con el fin de inducir variabilidad exógena en la inflación a través de terceras variables que la instrumenten, es decir, que estén correlacionadas con ella, pero sean exógenas. Veamos.

\section{ii. Variables instrumentales: instrumentando (débilmente) la inflación}

En una regresión por el método de las variables instrumentales, para que el instrumento utilizado sea válido deben cumplirse dos condiciones de exclusión: ser relevante (estar correlacionado con el predictor al cual instrumenta) y ser exógeno (no estar correlacionado con los residuos de la ecuación en la cual entra como instrumento).

Formalmente, siendo $X_{i}$ el regresor de interés, $Z_{i}$ la variable a utilizar como instrumento del mismo, y $u_{i}$ el termino residual de la ecuación estructural (en la cual predecimos nuestra variable de interés, $Y_{i}$ ), las condiciones de exclusión exigen que:

$$
\begin{array}{lll}
\operatorname{cov}\left(Z_{i}, X_{i}\right) \neq 0 & \rightarrow & \text { Instrumento relevante } \\
\operatorname{cov}\left(Z_{i}, u_{i}\right)=0 & \rightarrow & \text { Instrumento exógeno }
\end{array}
$$

Por ejemplo, Barro (2013) es consciente del problema de endogeneidad en su modelo y utiliza variables instrumentales para lidiar con él. El problema de los instrumentos utilizados es que difícilmente cumplan con las restricciones de exclusión que permiten aceptarlos como instrumentos válidos. Él mismo, en su ensayo, muestra evidencia de que la correlación entre la independencia del banco central (uno de los instrumentos seleccionados) y la tasa de inflación es sumamente baja.

\section{Primer instrumento débil: la inflación rezagada}

Más problemático es el caso de instrumentar la inflación con rezagos de sí misma. Por empezar, si la inflación es estacionaria, en el sentido de que oscila en torno al mismo 
valor a través del tiempo, instrumentar la inflación por su rezago es equivalente a instrumentar la inflación por su valor contemporáneo (lo que conduce al método de mínimos cuadrados ordinarios), conservando el problema de endogeneidad. ¿Por qué sucede esto? Si la inflación sigue un proceso estacionario, su media será independiente del dominio del tiempo. Para ilustrar esto, supóngase que la inflación sigue un camino aleatorio sin intercepto, tal que:

$$
\pi_{t}=\pi_{t-1}+e_{t}
$$

Donde $e_{t}$ es un choque aleatorio o ruido blanco, con varianza constante y media 0 . El valor esperado de la inflación, con esta estructura, será:

$$
E\left(\pi_{t}\right)=\sum_{i=0}^{\infty} E\left(e_{t-i}\right)=0
$$

Nótese que, al tomar una muestra finita — un subsegmento del dominio del tiempo, $T=(t-\infty, t]$ — el valor esperado de la media muestral será este valor independientemente del período tomado como muestra. Esto significa que el promedio muestral de la inflación contemporánea oscilará en torno al mismo valor que el promedio de la inflación con rezago. En estas condiciones, el instrumento será débil, porque la correlación entre el crecimiento económico y la inflación contemporánea, por un lado, y la inflación rezagada, por otro, será la misma, violando la condición de instrumento exógeno. En efecto, tomando una muestra de la tasa de variación del índice de precios al consumidor ${ }^{6}$ durante casi seis décadas (1961-2016) para 24 países calificados como emergentes por el $\mathrm{MSCI}^{7}$ y promediando los valores de todos los países para cada año, podría computarse un test de raíz unitaria para la serie resultante. Si el resultado del test implicara que ésta no fuese estacionaria, el problema señalado carecería de importancia para los mercados emergentes.

Realizando este procedimiento y efectuando el test aumentado de Dickey-Fuller para la siguiente especificación: $\Delta \pi_{t}=(\gamma-1) \pi_{t-1}+\varepsilon_{t}$ se rechaza la hipótesis nula de existencia de raíz unitaria, por lo que no hay evidencia muestral de que la serie no sea estacionaria $(D F \tau=-3.95, p<0,01)$.

Sin embargo, los mercados emergentes son países que han atravesado numerosos shocks externos, problemas fiscales y períodos de estabilización, por lo que la muestra contiene numerosos valores anómalos, u outliers. Identificando estos valores a partir del rango intercuartil ${ }^{8}$, excluyéndolos de la muestra y recalculando el estadístico de Dickey-Fuller,

\footnotetext{
6 Datos extraídos de la base de datos World Development Indicators del Banco Mundial, en adelante WDI. 7 La muestra incluye todos los países calificados como emergentes por MSCI excepto Taiwán, cuyos datos no están disponibles en WDI. En adelante, cuando se haga referencia a la calificación de MSCI se tratará de este grupo de países.

8 El método es el mismo utilizado para elaborar los conocidos diagramas de caja y bigote, o boxplots. Consiste en calcular 1,5 veces el rango intercuartil, y considerar como outliers a todos los valores que estén a una
} 
el nuevo valor obtenido no rechaza la hipótesis nula ( $D F \tau=-0,97, p>0,1)$. La evidencia obtenida, entonces, no es robusta a la presencia o ausencia de estos valores anómalos. Si se realiza el test de raíz unitaria una vez más, con la serie limpia de outliers y para el período 1995-2016, se logra rechazar la hipótesis nula $(D F \tau=-2,02, p<0,05)$, recuperando evidencia de que la serie es estacionaria.

Como se mencionó en la discusión de la sección 2, excluir outliers de una medición siempre es una decisión polémica. Los valores anómalos, en ciertas ocasiones, pueden brindar valiosa información y revelar patrones en los datos que de otra manera se perderían. Analizando la cantidad de observaciones clasificadas como outliers por período quinquenal, y la inflación promedio del período, se obtienen las siguientes estadísticas:

Cuadro 4. Inflación y outliers

\begin{tabular}{cccc}
\hline Quinquenio & Inflación promedio & Outliers & ${\text { Outliers }(\%)^{\mathrm{a}}}^{\mathrm{a}}$ \\
\hline 1960 & $12 \%$ & 3 & $3 \%$ \\
1965 & $30 \%$ & 4 & $3 \%$ \\
1970 & $22 \%$ & 3 & $2 \%$ \\
1975 & $23 \%$ & 6 & $5 \%$ \\
1980 & $28 \%$ & 15 & $12 \%$ \\
1985 & $90 \%$ & 18 & $15 \%$ \\
1990 & $241 \%$ & 19 & $16 \%$ \\
1995 & $20 \%$ & 11 & $9 \%$ \\
2000 & $7 \%$ & 2 & $2 \%$ \\
2005 & $6 \%$ & - & - \\
2010 & $5 \%$ & - & - \\
2015 & $5 \%$ & - & - \\
\hline
\end{tabular}

a. Porcentaje del total de observaciones del período, para 24 países MSCI.

Mencionamos que los mercados emergentes estuvieron sujetos a distintos shocks. Quizás el período más ilustrativo al respecto sean las crisis de deuda de la década de 1980, los colapsos fiscales asociados y los consiguientes programas de ajuste estructural de principios de la década de 1990. En los quinquenios de 1985 y 1990 la inflación promedio llegó al 90\% y 241\% anual, respectivamente. Justamente, en los quinquenios desde 1980 hasta 1995 es donde yace la mayor cantidad de outliers, que además representan una elevada proporción (entre el 12\% y el 16\%) del total de observaciones de esos períodos. En este contexto, eliminar outliers implica principalmente descartar utiliza el valor 6 en lugar del usual 1,5, dado que el nivel de asimetría de la serie de tiempo llevaría a que una gran proporción de los datos sea calificada como outlier. 
observaciones asociadas a crisis fiscales o shocks externos relacionados con la crisis de la deuda, que generaron aceleraciones inflacionarias muy por encima de la tasa de inflación habitual. La pregunta que surge inmediatamente es: jes esto correcto? En un contexto como el mencionado, los movimientos de la inflación son principalmente inducidos por los shocks, y no variabilidad exógena como la que necesitamos para evaluar su impacto en el crecimiento económico. En otras palabras, podrían ser movimientos endógenos, que capturan información de otras variables (términos de intercambio, movilidad internacional de capitales, crisis fiscal, etc.). Visualizar gráficamente estas observaciones en la serie temporal de inflación puede saldar definitivamente este punto:

Gráfico 6. Valores anómalos. Inflación anual por país, en logaritmo natural. 1961-2016

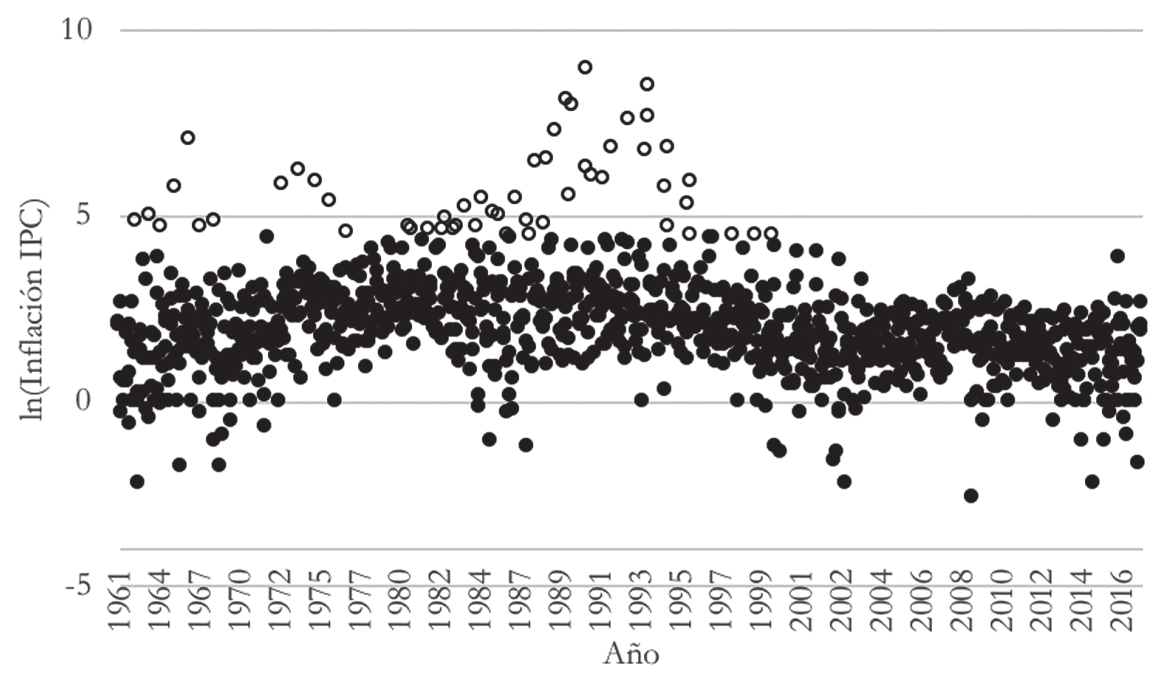

- Valor normal o Valor anómalo/Outlier

Elaboración propia. Logaritmo natural de la variación anual del Índice de Precios al Consumidor (Fuente: Banco Mundial, WDI) para la muestra de países MSCI. Cada punto representa el logaritmo de la inflación de un país en un año dado. Por imposibilidad de cálculo, 31 casos de variación del IPC negativa quedan excluidos.

Del gráfico anterior, expresado en términos logarítmicos, se desprende que la inflación sigue, en general, un camino aproximadamente estable, pero que a partir de 1980 (si bien ya había algunos valores anómalos anteriores), comienza a aparecer una cantidad cada vez mayor de observaciones inusualmente altas, alcanzando un pico y luego decayendo hacia mediados de la década de 1990. Para obtener una imagen más limpia, pueden promediarse las observaciones de todos los países para cada año, obteniéndose el siguiente gráfico: 
Gráfico 7. Valores anómalos. Inflación anual, promedio de países MSCI.

1961-2015

600

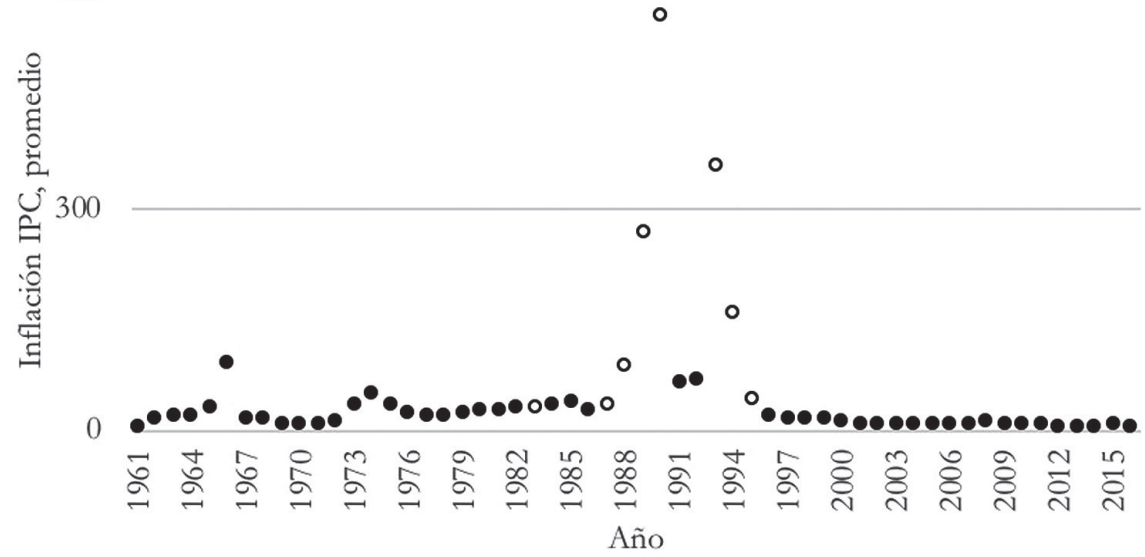

- Menos de $10 \%$ de valores anómalos o Más de 10\% de valores anómalos

Elaboración propia. Promedio por año de la variación del IPC (Fuente: Banco Mundial, WDI) para la muestra de países MSCI. Los puntos marcados en blanco son aquellos años en los cuales el 10\% o más de los países presentan una tasa de inflación identificada como un valor anómalo, u outlier.

Ya sea graficando todas las observaciones disponibles en logaritmos o promediando los valores anuales en niveles, se observa que los valores anómalos aparecen principalmente concentrados en una región específica de la serie y no dispersos a lo largo de todo el período muestral. Por lo tanto, mantenerlos en la muestra distorsionaría los datos, por lo que es admisible su remoción, si no con fines de inferencia, al menos con fines descriptivos.

Una vez removidos los outliers, la evidencia muestral no es decisiva respecto a si la inflación rezagada es un mal instrumento por problemas de estacionalidad. Pero, adicionalmente, estos datos permiten ver algo más: si se agrupan los datos en períodos de 2 años y se calcula la correlación entre estos promedios bianuales y el promedio bianual de, por ejemplo, tres períodos atrás, la correlación es de 0,56 . Si se aumenta el período de agregación a 3 y 4 años, esta correlación es 0,36 y 0,10 respectivamente. Esto nos introduce en otro problema, más general, que tiene la utilización de la inflación rezagada como variable instrumental.

La inflación es, con alta probabilidad, un fenómeno con memoria: shocks en un momento del tiempo afectan los valores futuros de la serie por un largo período (a través de expectativas adaptativas y mecanismos de indexación, y particularmente en países emergentes o en desarrollo). En el ámbito de las series temporales, esta propiedad se la denomina persistencia. Cuando una serie es persistente, existe correlación serial en la ecuación instrumental (aquella en la cual se regresa la variable $X_{i}$ con el instrumento 
$Z_{i}$ como predictor), y el instrumento es clasificado como instrumento débil. Al utilizar un instrumento débil, los coeficientes estimados en la ecuación en forma reducida (aquella en la cual se predice $Y_{i}$ ) tendrán una varianza amplia y, en muestras pequeñas (como las que se utilizan en los análisis de crecimiento, por la naturaleza misma de los datos), estarán sesgados.

El inconveniente señalado es un problema de series de tiempo, y las regresiones del crecimiento son, en parte, estudios de corte transversal, donde los datos temporales se promedian para cada país, de manera de tener un solo punto con la media del período para cada uno. No obstante, si la hipótesis de que la inflación tiene un impacto significativo en el crecimiento del producto es cierta, y los valores pasados de la inflación tienen una fuerte influencia durante largo tiempo en sus valores futuros, entonces los rezagos de la inflación utilizados como instrumentos estarán correlacionados con la tasa de crecimiento del producto, y no se cumplirá la segunda restricción de exclusión, esto es: el instrumento no será exógeno.

Si fuera cierto que la inflación rezagada y la inflación contemporánea brindan información similar, la correlación entre la inflación de un período y la de períodos anteriores debería ser alta. Podría, por ejemplo, calcularse el promedio de la inflación durante períodos de cinco ańos y el promedio de inflación durante los cinco ańos previos a cada período, y luego computarse el coeficiente de correlación entre ambas series.

Agrupando los años en períodos de distinta longitud, se observa una correlación moderada-alta que decrece cuando el criterio de agregación es un número mayor de años, extinguiéndose en el largo plazo. Sin embargo, removiendo los outliers y volviendo a computar esta correlación, el coeficiente obtenido oscila entre 0,54 y 0,84 , aún para períodos de agregación de nueve años:

Cuadro 5. Correlación entre la inflación de un período y el anterior

\begin{tabular}{ccc}
\hline Años agrupados & Muestra completa & Sin outliers \\
\hline 2 &, 745 &, 825 \\
3 &, 485 &, 837 \\
4 &, 404 &, 769 \\
5 &, 219 &, 759 \\
6 &, 360 &, 684 \\
7 &,- 056 &, 621 \\
8 &, 271 &, 628 \\
9 &,- 065 &, 540 \\
\hline
\end{tabular}

Elaboración propia, a partir de agrupar la serie calculando el promedio para cada grupo de años, y luego calculando la correlación con la misma serie rezagada en un período. 
La información del cuadro 5 sugiere que existe correlación entre la inflación de un período y la inflación de períodos anteriores. Esto, sin embargo, es insuficiente para visualizar la existencia de un problema de persistencia. Si recreásemos una ecuación instrumental regresando el promedio anual de la inflación (para todos los países de MSCI) contra rezagos de sí misma, podríamos analizar los residuos de la regresión correspondiente a través de un test de autocorrelación serial, cuyos resultados brindarían evidencia de que estados pasados de la serie influyen (o no) en sus estados futuros.

En primer lugar, utilizando un rezago, el test LM de Breusch-Godfrey rechaza la hipótesis nula de no autocorrelación de segundo y tercer grado en la serie promedio de inflación anual, con un $10 \%$ de significatividad $(\operatorname{LM}(1)=0,23, p=0,63 ; \operatorname{LM}(2)=$ $5,8, p=0,05 ; \operatorname{LM}(3)=6,4, p=0,09)$. Esta conclusión sobre la existencia de autocorrelación es robusta a la remoción del intercepto y a la adición de un rezago adicional de la inflación, aunque no a la remoción de outliers.

En segundo lugar, si este problema de la serie temporal fuese heredado de las regresiones de corte transversal, entonces debería observarse un resultado similar utilizando el promedio de inflación para un rango más amplio de años (como se hace típicamente en las regresiones de convergencia condicional o los modelos con umbrales y/o no lineales), el cual sería escogido como variable instrumental. Si se utiliza un promedio trianual (es decir, se toma la serie de inflación anual promedio de todos los países de MSCI y se promedian sus valores trianualmente) y se estima una regresión de la inflación con respecto a su valor rezagado en 1 período, se encuentra evidencia de auto correlación de primer orden $(\operatorname{LM}(1)=2,72, p=0,09)$. Si se utiliza, en cambio, su valor rezagado dos períodos, la evidencia aumenta hasta correlación serial de quinto orden inclusive. Si el período a promediar se extiende a cinco años esta evidencia se diluye, pero aún hay evidencia de autocorrelación de orden 3 si se remueven los outliers $(\operatorname{LM}(3)=6,79, p=0,07)$.

La evidencia, aunque lejos de ser conclusiva, no descarta la existencia de autocorrelación de distintos órdenes en la serie de tiempo de la inflación. Los datos, entonces, aportan cierta evidencia consistente con la existencia de persistencia y con que este problema afectaría el resultado de las regresiones de convergencia condicional que utilicen sus rezagos como instrumento. Reconocer este hecho arroja sombra sobre la consistencia de la estimación realizada por Barro (2013) para los fines de este ensayo.

\section{Segundo instrumento débil: el status colonial previo}

El siguiente instrumento que Barro (2013) utiliza es el estatus colonial previo. La nueva economía institucional — La Porta et al., 1997; Acemoglu et al., 2001; Engerman y Sokoloff, 1994) — ha aportado evidencia de que el evento histórico de la colonización impacta en el nivel de desarrollo de un país a través de su interacción con múltiples canales institucionales y geográficos (sistema legal heredado, carácter de las instituciones heredadas, dotación de recursos al momento de la conquista). Es implausible 
que el único impacto en la tasa de crecimiento actual de haber sido colonia sea a través del efecto de dicho evento en la tasa de inflación, por lo que el instrumento tampoco cumple las condiciones de exclusión, a menos que se tengan en cuenta todos los canales a través de los cuales impacta en la tasa de crecimiento actual. Esto último implicaría, en términos estadísticos, incluir una batería de variables índice que capten las diferencias institucionales entre economías. Estos índices, de los cuales existen varios (que, de hecho, se utilizan, por ejemplo, en Barro (2013)), son una medida cuantitativa de un aspecto (la institucionalidad) que tiene múltiples dimensiones cualitativas e informales, por lo que aun incluyendo estos controles debería luego lidiarse con problemas de error de medición en los mismos.

\section{Tercer instrumento débil: la independencia del banco central}

Hasta aquí hemos mostrado que ni los rezagos de la variable ni el status colonial previo son buenos instrumentos de la inflación. Retornemos al primer instrumento mencionado: la independencia del banco central, que es utilizado por Fischer (1993) y Cukierman et al. (1993), entre otros autores. Si bien Barro (2013) señala la baja correlación entre el índice de independencia del banco central (en adelante CBI, por su sigla en inglés central bank independence) y la tasa de inflación en su muestra (1960-1990), esto debe examinarse con mayor rigor.

En primer lugar, el índice de CBI de cada país no es una variable aislada, sino que es parte de su sistema institucional. Distintos países tienen distintos arreglos institucionales; más aún, países con instituciones formales similares, tienen resultados económicos diferentes, porque mantienen, por ejemplo, instituciones informales diferentes, o relaciones internacionales distintas. Por lo tanto, es de esperar que la correlación entre CBI e inflación esté afectada por una serie de heterogeneidades inobservables. En segundo lugar, lo dicho en el párrafo anterior implica que el índice de $\mathrm{CBI}$ es una variable que mide un aspecto de la institucionalidad de las economías, por lo cual está sujeto a los mismos problemas de medición mencionados en el punto 2 de esta sección.

Cukierman et al. (1993) señalan que existen diferencias entre la independencia legal del banco central y la independencia efectiva de dicha institución. Esta última es, precisamente, una heterogeneidad inobservable, cuyo efecto capturan a partir de un proxy que mide el tiempo que permanece en funciones el presidente del banco central (GTR, Governor Turnover Ratio por su sigla en inglés), y que reflejaría de forma más fidedigna su margen de maniobra para procurar la estabilidad de precios. Los autores encuentran evidencia muestral de que el GTR tiene poder explicativo suficiente como para ser un buen instrumento de la inflación en el caso de los países en vías de desarrollo. No se verifica la misma situación en el caso de los países desarrollados, lo que podría indicar que existen no linealidades en la muestra. 
Lo expuesto sugiere, por un lado, que probablemente la independencia del banco central sea el mejor instrumento disponible para estimar el efecto causal de la inflación en el crecimiento (sobre todo, porque podría tener poder explicativo para nuestro caso de interés, los mercados emergentes). Además, nos introduce a la discusión de las nolinealidades en esta relación, que consideran parte de los modelos escogidos en este trabajo para testear su validez externa e interna.

No obstante, GTR no es necesariamente un buen instrumento de la inflación: primero, no es necesariamente cierto que una baja rotación implique un mayor margen de maniobra: existen muchos casos de captura de la autoridad monetaria por parte del poder ejecutivo, donde puede observarse un presidente del banco central que permanece en el cargo, pero cuyo margen de maniobra es exiguo (determinado, por ejemplo, por la dominancia fiscal de la emisión monetaria). Segundo, y más grave, GTR no es una variable exógena, sino que puede haber simultaneidad: una alta inflación puede conducir a la destitución del presidente del banco central por una política monetaria fallida y acelerar la tasa de rotación en el cargo. Finalmente, es una variable de difícil medición y para la cual no se cuenta con una amplia base de datos. Por estos motivos, parece imponerse la utilización de la independencia del banco central de iure como instrumento de la inflación, tal como hace Barro.

El principal problema de este instrumento no parece ser teórico, sino muestral: su baja variabilidad. Barro (2013), tal como fue señalado, encuentra una baja correlación en su muestra entre CBI e inflación. La base de datos diseñada por Garrida (2016), que elabora un índice de CBI de iure similar al de Cukierman et al. (1993) con mayor cobertura geográfica y temporal, permite analizar si el hallazgo de Barro se sostiene en el caso de los mercados emergentes y con información actualizada. En efecto, la situación en este corte transversal de datos, a primera vista, es menos alentadora: primero, computando el desvío estándar para cada país como proporción del promedio y analizando su distribución por percentiles $\left(P_{25}=0,17, P_{50}=0,28, P_{75}=0,42\right)$, se observa que la mitad de los países tienen un nivel de variación inferior al 28\% de su media. Con independencia de que el instrumento sea teóricamente bueno, se necesitaría que posea suficiente variabilidad para que fuese útil en una regresión. Peor aún: la correlación entre CBI e Inflación, promediando para cada país el período 1970-2012, es positiva.

Sin embargo, es prematuro detenerse en el punto en el que se detiene Barro (2013), porque la base de datos muestra algo más: si en lugar de promediar todos los años para cada país, se promedian todos los países para cada ańo (de modo de obtener una serie temporal promedio), la correlación es fuertemente negativa. Gráficamente, pueden visualizarse las dos situaciones de la siguiente manera: 
Gráfico 8. Inflación vs. Independencia del Banco Central, 1970-2012

Panel a. Corte Transversal

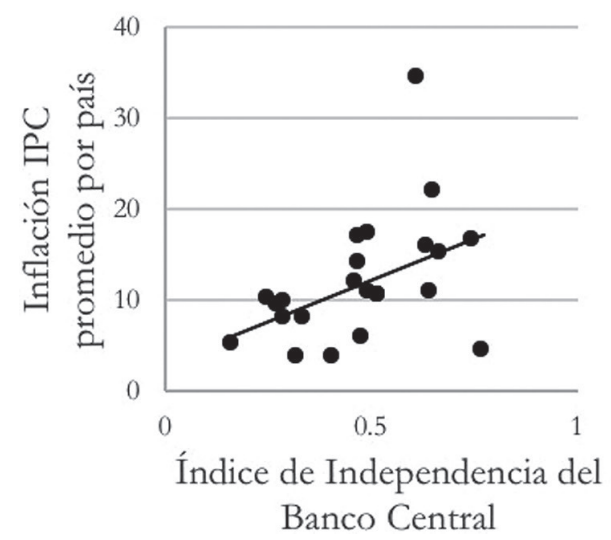

Panel b. Serie Temporal

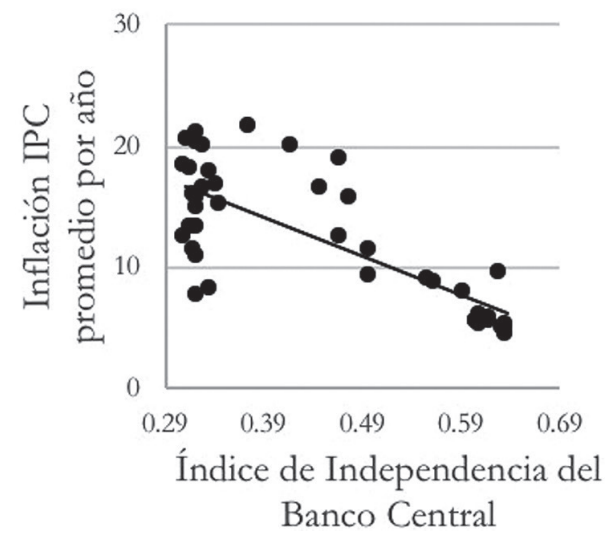

Elaboración propia en base a datos del Índice de Precios al Consumidor (Fuente: Banco Mundial, WDI) y un índice compuesto de Independencia del Banco Central a la Cukierman (Fuente: Garrida (2016)). El panel a (izquierda) muestra el promedio para cada país de MSCI en el período 1970-2012. El panel b (derecha) muestra el promedio para cada ańo de todos los países de la muestra.

¿Qué significa esta discrepancia en el coeficiente de regresión, según se analice la dimensión temporal o la dimensión individual? Cómo fue argumentado previamente para el caso de las instituciones, no existen razones para esperar que el efecto marginal del CBI sobre la inflación sea estable a través de los países (y tampoco a través del tiempo). Por lo tanto, pueden existir importantes efectos fijos en cada país y en cada período de tiempo (debido a diferencias en el arreglo institucional completo de la economía en cuestión), que se pierden al realizar una estimación por corte transversal. La pendiente positiva que se observa en ese caso podría ser en realidad una pendiente negativa afectada por heterogeneidades inobservables idiosincráticas de cada mercado.

De este análisis se deduce que el enfoque correcto, entonces, sería utilizar datos de panel, para explotar plenamente la información de la base de datos. Sin embargo, aún en dicho caso, la baja variabilidad del instrumento podría impedir una adecuada identificación. ¿Qué correlación entre inflación y CBI refleja un análisis de datos de panel? Utilizando el CBI, calculado por Garrida, para 23 de los mercados emergentes de MSCI, y añadiendo la variación del índice de precios al consumidor extraído de los WDI, se obtiene un panel desbalanceado con 831 observaciones entre 1970 y 2012. La hipótesis planteada de que los efectos (fijos) idiosincráticos de los países están correlacionados con el efecto marginal del CBI en la inflación implica que no son un choque aleatorio y que, entonces, si permanecen en el residuo, alterarían la estimación. Esto es porque, al estar correlacionados con la inflación y con la independencia del banco central, actuarían como una variable relevante omitida que, al no ser incluida en la estimación, generaría 
endogeneidad en el coeficiente estimado. En otros términos, es análogo a sostener que se rechazaría la hipótesis nula del test de Durbin-Wu-Hausman (véanse Durbin, 1954; Wu, 1973; o Hausman, 1978). En dicho test, la hipótesis nula es que un modelo de efectos aleatorios es consistente y eficiente, y la hipótesis alternativa es que sólo un modelo de efectos fijos sería consistente, ajustando este último mejor a los datos del panel.

En efecto, al aplicar este test al panel obtenido se rechaza la hipótesis nula $\left(\chi^{2}(1)=4,3, p=0,4\right)$ y, por lo tanto, tenemos evidencia de que existen efectos fijos estructurales en el panel. El resultado es aún más significativo si se remueven los outliers.

Regresando, entonces, la inflación contra el CBI con una estimación de efectos fijos, se obtiene el coeficiente esperado: el efecto marginal del CBI en la inflación es negativo y significativo para la muestra de mercados emergentes. Además, la magnitud de dicho coeficiente es económicamente relevante: aproximadamente -25 , lo que significa que por cada aumento del $10 \%$ en el índice de independencia del banco central, la inflación promedio anual desciende un 2,5\%. Removiendo outliers, el coeficiente es aún significativo, pero de una magnitud 10 veces inferior. Esta diferencia cuantitativa podría ser indicio de que existe un problema de simultaneidad similar al mencionado para el caso de GTR, que se revela en presencia de crisis inflacionarias y que produciría un sesgo en la estimación.

En conclusión: dejando de lado el problema de su baja variabilidad, la independencia de iure del banco central puede desempeñar el papel de instrumento válido de la inflación en las regresiones del crecimiento, si y solo sí se cumple con dos condiciones: Primero, que la estimación se realice con datos de panel (para aprovechar tanto la información de corte transversal como la dimensión temporal de los datos). Con esto, el índice de CBI se correlaciona con la inflación (y con el signo esperado): cumple con la condición de un instrumento relevante. Segundo, que en la estimación se utilicen variables de control apropiadas para mitigar el problema de simultaneidad y captar los otros canales por medio de los cuales la independencia del banco central y el crecimiento se correlacionan (como, por ejemplo, la calidad institucional de un país). En ausencia de dichos controles, el índice de CBI podría ser endógeno en la forma reducida de las regresiones de crecimiento. Pero, si se incluyen, se cumple con la segunda restricción de exclusión: el instrumento sería exógeno.

\section{iii. Endogeneidad por variables omitidas. Inflación, desarrollo financiero y crecimiento}

Otra fuente de endogeneidad posible está relacionada con la omisión de variables: el vínculo entre inflación y desarrollo financiero. Tal como fue mencionado anteriormente, Choi, Smith y Boyd (1996) explican que la inflación erosiona el nivel de desarrollo financiero de una economía. A su vez, el desarrollo financiero tiene un efecto causal positivo en el crecimiento económico, siendo el canal fundamental de ello las ganancias de productividad que habilita, al canalizar los fondos de los agentes superavitarios 
a proyectos riesgosos y más productivos que el promedio — ver Levine (2004), Pagano (1993)-.

En efecto, Levine y Zervos (1998), en un análisis de corte transversal, encuentran evidencia de que tanto la inflación como el desarrollo financiero tienen un efecto marginal independiente y significativo en el crecimiento económico. En cambio, Levine y Easterly (2001) realizan una estimación de datos de panel por el método generalizado de los momentos (GMM), en la cual detectan que el coeficiente que acompaña a la inflación pierde su significatividad estadística cuando se incluyen variables correlacionadas con el desarrollo financiero. En otras palabras: su trabajo aporta evidencia muestral de que la inflación no goza de un efecto marginal independiente en el crecimiento económico, sino que es el desarrollo financiero la variable que genera un efecto en este último; la inflación tendría sólo un impacto indirecto en la medida en que afecte a aquel.

Entonces, de acuerdo con Levine y Easterly (2001), la inflación afecta el crecimiento económico no directamente, sino a través de su efecto en el desarrollo financiero. Si este fuese el único canal de impacto de la inflación, en un diagrama causal se observaría:

$$
\begin{aligned}
& \pi_{t} \rightarrow D F_{t} \rightarrow g_{t}
\end{aligned}
$$

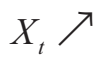

Siendo $D F_{t}$ un índice de desarrollo financiero. Este diagrama nos dice que las variables que tienen efecto directo sobre el crecimiento económico son el desarrollo financiero y otra serie de covariables aglomerados en $X_{t}$. La inflación, en cambio, tiene impacto en el desarrollo financiero, pero no tiene efecto directo en el crecimiento económico. La inflación y el crecimiento económico son independientes (de forma condicional a la presencia del desarrollo financiero), lo que convierte a la primera en una variable irrelevante.

Si este diagrama es cierto, significa que, si se estima una regresión en la cual no se incorpora un proxy del nivel de desarrollo financiero, o el que se incorpora no es suficiente para capturar todo el efecto de la inflación en dicha variable, el coeficiente que acompañe a la inflación en la estimación resultante tendrá un componente espurio: capturará variabilidad que es inducida en el crecimiento económico no por la inflación misma, sino por el desarrollo financiero.

Ahora bien, en un caso más general, podría suceder que la inflación tenga efecto tanto en el desarrollo financiero como en el crecimiento económico, tal como estiman Levine y Zervos (1998), y que exista una tercera variable que afecte a ambos predictores. Supongamos, por ejemplo, que la confianza en la moneda lleva a un mayor nivel de demanda de dinero y a una mayor disponibilidad a celebrar contratos formales, afectando tanto a la inflación como al desarrollo financiero. En este caso, ambas variables estarían vinculadas por una heterogeneidad inobservable, tal que: 


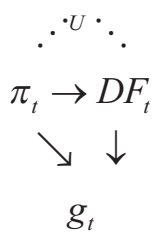

¿Qué revela este diagrama causal? Tanto la inflación como el desarrollo financiero tienen un efecto directo en el crecimiento económico. A su vez, la inflación tiene un impacto en el desarrollo financiero, y, finalmente, ambas variables están correlacionadas por una heterogeneidad inobservable $(U)$. El desarrollo financiero cumple con el teorema del Back Door (véase en Pearl, 2000): su presencia en la regresión permite captar el efecto causal directo de la inflación en el crecimiento (la flecha que va de $\pi_{t}$ a $g_{t}$ ). Pero, en este caso más general, no incluirlo como covariable implica la omisión de variables relevantes: ante su ausencia, existe una relación de dependencia mutua entre la inflación y el crecimiento causada por $U$, generándose un problema de endogeneidad (los movimientos en la inflación no serán exógenos, sino que estarán correlacionados con los residuos de la regresión). Aun cuando la inflación tiene un efecto directo causal en el crecimiento económico, la omisión del desarrollo financiero distorsionará la estimación de dicho impacto.

El problema de la literatura revisada y analizada en este trabajo es que, con excepción de Levine y Easterly (2001) y Levine y Zervos (1998), ninguno de los siete artículos restantes incorpora medidas del desarrollo financiero como variable explicativa. De allí la posibilidad de un sesgo de endogeneidad en las respectivas regresiones y la menor validez interna relativa de los modelos asociados.

\section{INFLACIÓN Y CRECIMIENTO EN LOS MODELOS DE CONVERGENCIA CONDICIONAL: EL CASO DE PERÚ}

A lo largo del último medio siglo, Perú ha atravesado períodos de alta inflación e inestabilidad, cambios estructurales, y un largo período de una macroeconomía ordenada y un índice de precios estable. Esquemáticamente, la inflación en Perú, capturada en el siguiente gráfico (en escala logarítmica), podría dividirse en cuatro períodos: uno de inflación moderada y creciente, desde 1970 hasta aproximadamente 1978, cuando supera por primera vez el 50\% anual; un período de inflación creciendo exponencialmente que culmina en los episodios hiperinflacionarios de fines de la década de 1980 y principios de la década de 1990; un período de estabilización e inflación a la baja, hasta fines de la década de 1990; finalmente, un período de estabilidad e inflación baja desde 1999, cuando la evolución anual del IPC es por primera vez inferior al 5\%, hasta la actualidad. 
Gráfico 9. Inflación en Perú 1978-2016, en escala logarítmica

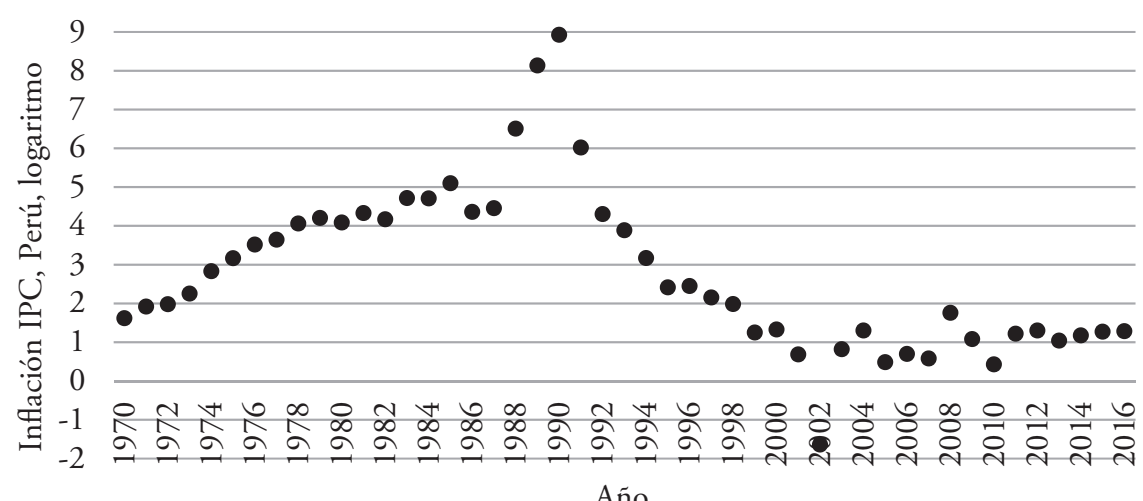

Elaboración propia en base a datos del Índice de Precios al Consumidor (Fuente: Banco Mundial, WDI).

La evolución dispar del nivel general de precios en Perú servirá para ilustrar las fortalezas y limitaciones de los modelos bajo análisis. En efecto, el análisis realizado en el gráfico 5 muestra que, en el caso de Perú, los modelos que predicen el comportamiento de la inflación en 1995-2016 con un menor error relativo son los de Fischer y Barro, dos modelos lineales. El modelo de Fischer, particularmente, predice con RMSE del 0.0033. Si ampliamos el período a predecir e introducimos datos desde 1978 a 2016, el RMSE en la tasa de crecimiento predicha por el modelo de Fischer pasa a ser 0,058. Este error, de un orden de magnitud muy superior al calculado previamente, se origina en una fuerte subestimación del crecimiento en Perú por parte del modelo: entre 1978-2016, la tasa de crecimiento de Perú centrada en torno a la media global fue de $-1,2 \%$, mientras que la tasa predicha para el mismo período por el modelo de Fischer es -7\%, resultando en una subestimación del crecimiento de 5,8\% en términos absolutos. En otras palabras: entre 1978-2016, el modelo de Fischer predice una tasa de crecimiento 5,8\% inferior al observado (en términos absolutos).

Los motivos del incremento en el error de predicción expuesto en el párrafo anterior pueden observarse descomponiéndolo para cada uno de los años que componen la muestra. En el siguiente gráfico, en el cual se muestra (para el período 1978-2016) cada uno de los valores observados de tasa de crecimiento (centrados en torno a su media) y el valor predicho por el modelo de Fisher ${ }^{9}$, se observa claramente que el mayor desvío en la predicción se produce en 1989-1990, años de hiperinflación.

\footnotetext{
9 Al utilizar observaciones puntuales para cada año en lugar de promedios para el período, emerge un problema de valores faltantes en algunas observaciones de algunas variables. Para poder continuar con el análisis, se optó por imputar el valor inmediatamente anterior o posterior disponible o, en el caso de que haya valores tanto anteriores como posteriores, utilizar el promedio de ambos.
} 
Gráfico 10. Crecimiento en Perú 1978-2016, real versus predicho por Fischer

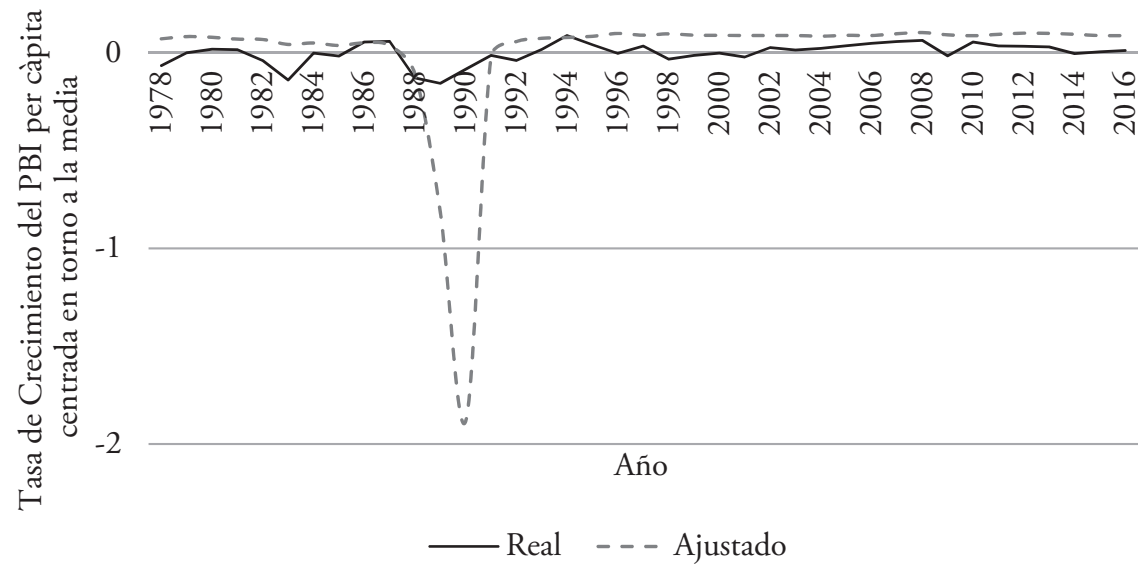

Elaboración propia. La linea "Real" refleja datos del PBI per cápita a valores PPA constantes de 2011 (Fuente: Banco Mundial, WDI), centrado en torno a la media del período. La linea "Ajustado" muestra los valores predichos al aplicar los coeficientes de Fischer (1993) a los valores de las variables de cada ańo.

En 1989-1990, el crecimiento predicho es muy inferior al observado. Esto es: ante valores extremos de la inflación, el modelo de Fischer no es adecuado para explicar el crecimiento de Perú. Esta notoria caída de la capacidad predictiva del modelo es ilustrativa de las virtudes y defectos de los modelos discutidos a lo largo de este trabajo. Tal como fue analizado, el modelo de Fischer se caracteriza por su linealidad y por su parsimonia (utilizar pocos predictores). Esto, que en condiciones macroeconómicas "normales" le permite generalizarse y predecir adecuadamente diversos escenarios, se transforma en una debilidad cuando se lo enfrenta a contextos extremos caracterizados por inestabilidad y valores atípicos en alguna de las variables, porque el modelo no puede captar las no-linealidades y los efectos de períodos de amplia variabilidad y cambios estructurales como los enfrentados por Perú.

Esto se materializa en dos situaciones: por un lado, la omisión de variables relevantes (fundamentalmente, el "desarrollo financiero") le impiden capturar información relevante para predecir el crecimiento. A modo de hipótesis, podría sostenerse que el desarrollo financiero se ve fuertemente afectado por episodios de hiperinflación (por ejemplo, por el rompimiento de contratos y una abrupta caída del crédito al sector privado), así como por los procesos de reforma estructural como los experimentados en los países latinoamericanos durante la década de 1990. Esto significa que las variables relacionadas con este aspecto de la economía contienen información relevante para el período, y que al omitirlas se genera una endogeneidad que distorsiona el coeficiente estimado para la inflación en el crecimiento. Por otro lado, al ser estos coeficientes lineales, el impacto de una tasa de inflación de, por ejemplo, el 4000\%, sería el doble que de una del 2000\%, cuando lo esperable es que el mayor daño de un proceso hiperinflacionario (rompimiento de contratos, 
indexación y retraso de otras variables macroeconómicas, cambios en la conducta de los agentes económicos, pérdida de la función informativa del sistema de precios) se produzca al ingresar en dicho régimen y que, por lo tanto, el impacto marginal de incrementos adicionales en la inflación no tengan un impacto similar.

\section{RESUMEN Y CONCLUSIONES}

El presente trabajo tuvo por objetivo aprehender la relación estructural entre la inflación y el crecimiento económico, y su manifestación en el caso de los países calificados como mercados emergentes según el índice MSCI de Morgan Stanley.

Comenzamos el estudio con un repaso del estado del conocimiento teórico sobre el vínculo entre la inflación y el crecimiento. Distintos autores presentan distintas posturas, según las cuales la inflación puede ser positiva (aumenta la rentabilidad relativa de la inversión, relaja las rigideces nominales), negativa (acorta los horizontes de inversión, desincentiva el crédito al sector privado, distorsiona las señales de precios) o incluso neutral (si los agentes tienen expectativas racionales y no existen fallas de mercado). Considerados en su conjunto, estos elementos parecen describir una relación no lineal en la cual el efecto sobre el crecimiento depende del nivel de inflación considerado y de la configuración de la economía en cuestión.

Posteriormente, procedimos en la segunda sección a analizar la validez externa de un conjunto representativo de investigaciones empíricas existentes: qué tan bien se generalizan sus conclusiones como para aplicarlos a una situación nueva, el crecimiento de las últimas dos décadas en los mercados emergentes. Detectamos algunas fortalezas y debilidades en estas simulaciones, la cuales nos dejaron importantes lecciones: la importancia de la relación entre desarrollo financiero e inflación, de la especificación y de la parsimonia en los modelos econométricos (para permitir su generalización).

Luego de dar cuenta de esto y levantar una señal de cautela sobre la validez externa de estos modelos econométricos (predicción), hicimos un repaso de las principales investigaciones empíricas existentes, enfocándonos en los inconvenientes que estos estudios tenían para identificar correctamente el efecto de la inflación en el crecimiento. Esta relación presenta endogeneidad (por simultaneidad, dependencia mutua y variables omitidas) y los instrumentos disponibles para dar cuenta de ella son escasos y débiles.

Las principales conclusiones de este trabajo son que:

- La relación negativa entre inflación y crecimiento se sostiene de manera generalizada en las dos variedades de modelos analizadas, en el tiempo y para distintas muestras. Sin embargo, en el trabajo testeamos la validez externa (predicción) e interna (robustez y propiedades de los estimadores obtenidos en las regresiones).

- Los modelos lineales y más parsimoniosos predicen mejor el crecimiento a partir de la inflación en muestras diversas de países y períodos. La parsimonia, si bien es una propiedad deseable, no debe llevar a omitir variables relevantes en la estimación. 
- Los modelos no lineales y con umbrales generan errores de predicción del crecimiento económico mayores, y algunos tienden a subestimar sistemáticamente (Kremer et al., 2011) o sobreestimar el crecimiento económico (Bick, 2010)). Asimismo, como la inflación descendió en el mundo en las últimas dos décadas a niveles inferiores a los umbrales, el poder predictivo de estas estimaciones econométricas tiende a perder peso.

- Entonces, dada la validez externa relativa de los modelos lineales, de convergencia condicional, estos siguen siendo mejor que el resto.

- Sin embargo, la repetición del ejercicio de simulación con una muestra acotada a la última década, período en el cual la inflación se mantuvo baja y estable, penaliza diferencialmente más (en términos de PRMSE) a algunos de los modelos lineales, lo cual podría ser un indicio de que la verdadera relación estructural subyacente entre inflación y crecimiento presenta no linealidades. Aun así, los modelos lineales siguen siendo aquellos que predicen mejor, y por lo tanto aquellos con mayor validez externa.

- La validez interna de los modelos está jaqueada por problemas de sesgos de endogeneidad, la presencia de outliers y especificación incorrecta de modelos (corte transversal en lugar de paneles). Queda, para investigaciones futuras, analizar cómo posibles errores de medición de la inflación también pueden generar sesgos y subestimaciones del verdadero impacto de la inflación en el crecimiento.

Respecto al sesgo por endogeneidad, las razones principales que argumentamos y probamos con datos propios y de otros trabajos son que:

- Aunque la verdadera relación entre la inflación y la tasa de crecimiento es nula si el dinero es superneutral, a raíz de un problema de simultaneidad es posible observar una correlación negativa entre ambas variables, porque el coeficiente estimado de la inflación podría estar capturando variabilidad endógena, inducida a través del efecto de otras variables.

- Los instrumentos utilizados por la literatura no cumplen con las condiciones de exclusión necesarias para una adecuada identificación del efecto causal de la inflación en el crecimiento.

- De éstos, el instrumento teóricamente más adecuado, la independencia del banco central, presenta una baja variabilidad muestral, lo que atenta contra la correcta estimación econométrica.

- Existen importantes diferencias estructurales entre países, que conducen a que las regresiones de corte transversal omitan importantes efectos fijos entre países y a lo largo del tiempo.

- La inflación tiene profundas interacciones con otras dimensiones de la economía, principalmente con aquellas que hacen al desarrollo financiero. La omisión de variables relacionadas a aquel último conduce a estimaciones que capturan variabilidad endógena, y por lo tanto distorsionan el efecto de la inflación en el crecimiento económico. 


\section{ANEXO}

La base de datos utilizada en el presente trabajo consta de 33 variables para 24 países (todos los países MSCI excluyendo Taiwán) para un período de hasta 57 años (1960-2016). Se trata de un panel con 31636 observaciones. Considerando la dimensionalidad del mismo $(33 \times 24 \times 57=45144)$ se notará que el panel está desbalanceado: tiene casi un 30\% de valores nulos. A continuación, se expone un resumen de la información contenida ${ }^{10}$ :

\begin{tabular}{|c|c|c|c|c|c|c|}
\hline Variable & $\mathrm{N}$ & Media & Desv. est. & $\operatorname{Pctl}(25)$ & Mediana & $\operatorname{Pctl}(75)$ \\
\hline Black Market Premium & 727 & 354,6 & 5,368 & 1,4 & 7,4 & 30,0 \\
\hline Superávit financiero público & 664 & $-2,4$ & 3,8 & $-4,4$ & $-2,3$ & $-0,2$ \\
\hline CBI & 882 & 0,5 & 0,2 & 0,3 & 0,4 & 0,6 \\
\hline Golpes de Estado & 1291 & 0,04 & 0,2 & 0 & 0 & 0 \\
\hline Crédito Privado a PBI & 1123 & 39,9 & 30,6 & 19,3 & 29,5 & 49,8 \\
\hline Crecim. PBI pc (\%) & 1142 & 2,6 & 4,5 & 0,8 & 3,0 & 5,1 \\
\hline Crecim. Población (\%) & 1368 & 2,0 & 2,3 & 0,8 & 1,7 & 2,5 \\
\hline Escolaridad femenina (\%) & 872 & 99,8 & 16,6 & 96,4 & 102,2 & 108,0 \\
\hline Fertilidad & 1344 & 3,4 & 1,7 & 2,0 & 2,8 & 4,9 \\
\hline Gasto público educación (\%) & 546 & 3,7 & 1,3 & 2,7 & 3,7 & 4,7 \\
\hline Ingresos del gobierno & 704 & 21,9 & 8,5 & 15,5 & 20,4 & 27,0 \\
\hline Consumo del gobierno (\%) & 1138 & 13,3 & 4,4 & 10,2 & 12,0 & 16,4 \\
\hline PBI per cápita (PPA 2011) & 637 & 18868 & 23575 & 7479 & 12370 & 20266 \\
\hline PBI per cápita (PPA 1996) & 23 & 7068 & 4258 & 3969 & 6674 & 8458 \\
\hline Deflactor PBI & 1137 & 41,5 & 272,1 & 3,6 & 7,8 & 16,0 \\
\hline Inversión a PBI & 1097 & 22,4 & 6,6 & 18,0 & 21,5 & 25,7 \\
\hline IPC & 1112 & 43,1 & 319,8 & 3,4 & 6,9 & 14,7 \\
\hline Expectativa de vida & 1344 & 66,5 & 7,7 & 61,4 & 68,2 & 71,9 \\
\hline Escolaridad masculina & 872 & 105,0 & 11,7 & 99,0 & 103,8 & 110,6 \\
\hline Calidad de instit. políticas & 1014 & 4,2 & 1,9 & 2 & 4 & 6 \\
\hline Población & 1076 & 136,3 & 272,2 & 18,1 & 42,2 & 91,1 \\
\hline Escolaridad primaria & 983 & 102,6 & 13,0 & 97,6 & 103,2 & 109,0 \\
\hline PBI (PPA 2011) & 1076 & 519065 & 1043064 & 100827 & 220979 & 505959 \\
\hline Imperio de la Ley & 432 & 0,1 & 0,7 & $-0,5$ & 0,004 & 0,6 \\
\hline Escolaridad secundaria & 912 & 69,7 & 24,8 & 50,6 & 74,3 & 91,5 \\
\hline Términos de Intercambio & 703 & 112,4 & 35,4 & 94,2 & 103,1 & 120,1 \\
\hline Apertura comercial & 1170 & 47,8 & 34,1 & 25,0 & 38,6 & 60,7 \\
\hline Turnover a GDP & 635 & 23,3 & 32,8 & 4,7 & 10,8 & 30,0 \\
\hline Guerras & 1368 & 0,5 & 0,9 & 0 & 0 & 1 \\
\hline Años de escolaridad & 1056 & 6,5 & 2,9 & 4,3 & 6,5 & 8,4 \\
\hline Años de secundaria & 1056 & 2,0 & 1,1 & 1,1 & 1,9 & 2,8 \\
\hline Ańos de terciario & 1056 & 0,4 & 0,3 & 0,2 & 0,3 & 0,5 \\
\hline
\end{tabular}

${ }^{10}$ La base de datos puede descargarse, junto con un detalle de la descripción y fuente de cada variable, del siguiente enlace: https:/www.dropbox.com/s/974a6hm6w5xxnsb/Fucci\%20\%26\%20Grandes\%20-\%20 Inflacion $\% 20 \mathrm{y} \% 20$ crecimiento $\% 2 \mathrm{C} \% 20$ dataset.xlsx?dl=0 


\section{REFERENCIAS BIBLIOGRÁFICAS}

Acemoglu, D., S. Johnson y J. Robinson (2001). The Colonial Origins of Comparative Development: An Empirical Investigation. American Economic Review, 91(5), 1369-1401. https://doi.org/10.1257/aer.91.5.1369

Baglan, D. y E. Yoldas (2014). Non-linearity in the Inflation-Growth Relationship in Developing Economies: Evidence from a Semiparametric Panel Model. Economics Letters, 125(1), 93-96. https://doi.org/10.1016/j.econlet.2014.08.015

Barreto, H. y F. Howland (2006). Introductory Econometrics using Monte Carlo Simulation with Microsoft Excel . Cambridge, UK: Cambridge University Press. https://doi.org/10.1017/ CBO9780511809231

Barro, R. (1991). Economic Growth in a Cross-Section of Countries. The Quarterly Journal of Economics, 106(2), 407-443. https://doi.org/10.2307/2937943

Barro, R. (2013) [1995]. Inflation and Economic Growth. Annals of Economics and Finance, 14(1), 121-144.

Bick, A. (2010), Threshold Effects of Inflation on Economic Growth in Developing Countries. Economics Letters, 108(2), 126-129. https://doi.org/10.1016/j.econlet.2010.04.040

Bittencourt, M. (2012). Inflation and economic growth in Latin America: Some panel timeseries evidence. Economic Modelling, 29(2), 333-340. https://doi.org/10.1016/j. econmod.2011.10.018

Briault, C. (1995). The Costs of Inflation. Bank of England Quarterly Bulletin, 35.

Choi, S., B. Smith y J. Boyd (1996). Inflation, Financial Markets and Capital Formation. Federal Reserve Bank of St. Louis Review, 78(3), 9-35. https://doi.org/10.20955/r.78.9-35

Cukierman, A., P. Kalaitziadakis, L. Summer y S. Webb (1993). Central Bank Independence, Growth, Investment, and Real Rates. Carnegie-Rochester Conference Series on Public Policy, 39(1), 95-140. https://doi.org/10.1016/0167-2231(93)90005-H

De Gregorio, J. (1996). Inflation, growth, and central banks: theory and evidence. Policy Research Working Paper Series, The World Bank, 1575.

Devlin, Robert y Ricardo Ffrench-Davis (1994). The great Latin American debt crisis: a decade of asymmetric adjustment, Sede de la CEPAL en Santiago (Estudios e Investigaciones), Naciones Unidas Comisión Económica para América Latina y el Caribe (CEPAL).

Durbin, J. (1954). Error in variables. Review of the International Statistical Institute, 22(1/3), 23-32. https://doi.org/10.2307/1401917

Easterly, W. y M. Bruno (1998). Inflation Crises and Long-Run Growth. Journal of Monetary Economics, 41(1), 3-26. https://doi.org/10.1016/S0304-3932(97)00063-9

Engerman, S. y K. Sokoloff (1994). Factor Endowments, Institutions and Differential Paths of Growth among New World Economies: a view from economic historians of the United States. En S. Harber (ed.), How Latin America Fell Behind (pp. 260-304). Stanford: Stanford University Press. https://doi.org/10.3386/h0066

Fanelli, J. y R. Frenkel (1996). Estabilidad y estructura: interacciones en el crecimiento económico. En J. Katz (ed.), Estabilización macroeconómica, reforma estructural y comportamiento industrial. Estructura y funcionamiento del sector manufacturero latinoamericano en los años 90. Buenos Aires: CEPAL/IDRC-Alianza Editorial.

Fischer, S. (1993). The Role of Macroeconomic Factors in Growth. Journal of Monetary Economics, 32(3), 485-512. https://doi.org/10.1016/0304-3932(93)90027-D

Garrida, A. (2016). Central Bank Independence in the World: A New Data Set. International Interactions, 42(5), 849-868. https://doi.org/10.1080/03050629.2016.1188813 
Hausman, J. (1978). Specification Tests in Econometrics. Econometrica, 46(6), 1251-1271. https://doi.org/10.2307/1913827

Hansen, B. (1999). Threshold Effects in Non-Dynamic Panels: Estimation, Testing, and Inference. Journal of Econometrics, 93(2). https://doi.org/10.1016/S0304-4076(99)00025-1

Jones, L. y Rodolfo E. Manuelli (1995). Growth and the effects of inflation. Journal of Economic DynamicsandControl, 19(8), 1405-1428.https://doi.org/10.1016/0165-1889(94)00835-6

Kocherlakota, N. (1996). Inflation and Growth - Commentary. Review of the Federal Reserve Bank of St.Louis, issue 1996(5), 170-172.

Kremer, S., A. Bick y D. Nautz (2011). Inflation and growth: new evidence from a dynamic panel threshold analysis. Empirical Economics, 44(2), 861-878. https://doi.org/10.1007/ s00181-012-0553-9

La Porta, R., F. Lopez-de-Silanes, A. Shleifer y R. Vishny (1997). Legal Determinants of External Finance.JournalofFinance, 52(3), 1131-1150.https://doi.org/10.1111/j.1540-6261.1997. tb02727.x

Levine, R. (2004). Finance and Growth: Theory and Evidence. En P. Aghion y S. Durlauf (eds.), Handbook of Economic Growth (vol. 1A, chapter 12, pp. 865-934). Elsevier. https://doi. org/10.3386/w10766

Levine, R. y W. Easterly (2001). It's Not Factor Accumulation: Stylized Facts and Growth Models. The World Bank Economic Review, 15(2), 177-219. https://doi.org/10.1093/ wber/15.2.177

Levine, R. y Zervos, S. (1998). Stock Markets, Banks, and Economic Growth. American Economic Review, 88(3), 537-558.

Lucas Jr., R. (1973). Some International Evidence on Output-Inflation Tradeoffs. American Economic Review, 63(4), 326-334.

Mankiw, N., D. Romer y D. Weil (1992). A Contribution to the Empirics of Economic Growth. Quarterly Journal of Economics, 107(2), 407-437. https://doi.org/10.2307/2118477

Moreno-Brid, J.C, J.C. Rivasy F. Villarreal (2014). Inflación y crecimiento económico. Investigación Económica, 73(290), 3-23. https://doi.org/10.1016/S0185-1667(15)30006-0

Pagano, M. (1993). Financial Markets and Growth. European Economic Review, 37(2-3), 613-622. https://doi.org/10.1016/0014-2921(93)90051-B

Pearl, J. (2000). Casuality: Models, Reasoning and Inference. Nueva York: Cambridge University Press.

Rodrik, D. (2008). The New Development Economics: We Shall Experiment, but How Shall We Learn? Working Paper Series, Harvard University, John F. Kennedy School of Government. https://doi.org/10.2139/ssrn.1296115

Sala-i-Martin, X. (1997). I Just Ran Two Million Regressions. American Economic Review, 87(2), 178-183. https://doi.org/10.3386/w6252

Sidrauski, M. (1990). Rational choice and patterns of growth in a monetary economy. American Economic Review, 57(2), 534-544. https://doi.org/10.1086/259552

Solow, R., \& Orphanides, A. (1990). Money, inflation and growth. En B. M. Friedman y F. H. Hahn (eds.), Handbook of Monetary Economics, vol. 1, chapter 6, Elsevier. https://doi. org/10.1016/S1573-4498(05)80009-8

Stockman, A. C. (1981). Anticipated inflation and the capital stock in a cash in-advance economy. Journal of Monetary Economics, 8(3). https://doi.org/10.1016/0304-3932(81)90018-0

Thirlwall, A. y C. Barton (1971). Inflation and Growth: The International Evidence. Banca Nazionale del Lavoro Quarterly Review, 24(98), 263-275. 
Tobin, J. (1965). Money and Economic Growth. Econometrica, 33(4), 671-684. https://doi. org/10.2307/1910352

Vegh, Carlos A., Luis Morano, Diego Friedheim, Diego Rojas (2017). Between a Rock and a Hard Place: The Monetary Policy Dilemma in Latin America and the Caribbean. LAC Semiannual Report; October 2017. Washington, DC: World Bank. https://doi.org/10.1596/28443

Wu, De-Min (1973). Alternative Tests of Independence between Stochastic Regressors and Disturbances. Econometrica, 41(4), 733-750. https://doi.org/10.2307/1914093

Documento recibido el 20 de diciembre de 2018 y aprobado el 26 de febrero de 2019 